\title{
Simultaneous reduction to normal forms of commuting singular vector fields with linear parts having Jordan blocks
}

\author{
Todor Gramchev* and Masafumi Yoshino ${ }^{\dagger}$ \\ Dipartimento di Matematica, Università di Cagliari \\ via Ospedale 72, 09124 Cagliari, Italy \\ e-mail: todor@unica.it \\ Graduate School of Sciences, Hiroshima University \\ Higashi-Hiroshima, 739-8526, Japan \\ e-mail: yoshino@math.sci.hiroshima-u.ac.jp
}

April 18, 2005

\begin{abstract}
We study a simultaneous linearizability of $d$-actions (and the corresponding $d$-dimensional Lie algebras) defined by commuting singular vector fields in $\mathbb{C}^{n}$ fixing the origin with a nontrivial Jordan block in the linear parts. We prove the analytic convergence of a formal linearizing transformation under a certain invariant geometric condition (cone condition) for the spectrum of $d$ vector fields generating a Lie algebra. (cf. Example 1.4.) If the condition fails, then we show the existence of divergent solutions of an overdetermined system of linearized homological equations. In a smooth category, the situation is
\end{abstract}

\footnotetext{
*Supported by NATO grant PST.CLG.979347, and GNAMPA-INDAM, Italy.

${ }^{\dagger}$ Supported by Grant-in-Aid for Scientific Research (No. 14340042), Ministry of Education, Science and Culture, Japan and GNAMPA-INDAM, Italy.
} 
completely different. We will show Sternberg's theorem for a commuting system of vector fields with a Jordan block although they do not satisfy a cone condition.

Key words: singular vector field, linearization, Jordan block, homological equation, Diophantine conditions, Gevrey spaces, decomposition

MSC classification: 37C05, 37F50, 37G05

\section{Simultaneous linearization}

Let $\mathbb{K}$ be $\mathbb{K}=\mathbb{C}$ or $\mathbb{K}=\mathbb{R}$, and $B=\infty, B=\omega$ or $B=k$ for some $k>0$. Let $\mathcal{G}_{B}^{n}$ denotes a $d$-dimensional Lie algebra of germs at $0 \in \mathbb{K}^{n}$ of $C^{B}$ vector fields vanishing at 0 . Let $\rho$ be a germ of singular infinitesimal $\mathbb{K}^{d}(d \geq 2)$ actions of class $C^{B}$

$$
\rho: \mathbb{K}^{d} \longrightarrow \mathcal{G}_{B}^{n} .
$$

We denote by $A c t^{B}\left(\mathbb{K}^{d}: \mathbb{K}^{n}\right)$ the set of germs of singular infinitesimal $\mathbb{K}^{d}$ actions of class $C^{B}$ in $0 \in \mathbb{K}^{n}$. By choosing a basis $e_{1}, \ldots, e_{d} \in \mathbb{K}^{n}$, the infinitesimal action can be identified with a $d$-tuple of germs at 0 of commuting vector fields $X^{j}=\rho\left(e_{j}\right), j=1, \ldots, d$ (cf. [10], [21]). We can define, in view of the commutativity relation, the action

$$
\begin{aligned}
\tilde{\rho}: & \mathbb{K}^{d} \times \mathbb{K}^{n} \longrightarrow \mathbb{K}^{n}, \\
\tilde{\rho}(s ; z) & =X_{s_{1}}^{1} \circ \cdots \circ X_{s_{d}}^{d}(z)=X_{s_{\sigma_{1}}}^{\sigma_{1}} \circ \cdots X_{s_{\sigma_{d}}}^{\sigma_{d}}(z), \quad s=\left(s_{1}, \ldots, s_{d}\right),
\end{aligned}
$$

for all permutations $\sigma=\left(\sigma_{1}, \ldots, \sigma_{d}\right)$ of $\{1, \ldots, d\}$, where $X_{t}^{j}$ denotes the flow of $X^{j}$. We denote by $\rho_{\text {lin }}$ the linear action formed by the linear parts of the vector fields defining $\rho$.

We shall investigate the necessary and sufficient condition for the linearization of $\rho$, namely, whether there exists a $C^{B}$ diffeomorphism $g$ preserving 0 such that $g$ conjugates $\tilde{\rho}$ and $\tilde{\rho}_{\text {lin }}$

$$
\tilde{\rho}(s ; g(z))=g\left(\widetilde{\rho_{\text {lin }}}(s, z)\right), \quad(s, z) \in \mathbb{K}^{d} \times \mathbb{K}^{n} .
$$

We recall that in [10], [17], [28] the linear parts were supposed to be diagonalizable, while in [32] the existence of $n-d$ anlalytic first integrals was 
required. (See also [1], [16]). Following Katok's argument in [21], we take a positive integer $m \leq n$ such that $\mathbb{K}^{n}$ is decomposed into a direct sum of $m$ linear subspaces invariant under all $A^{\ell}=\nabla X_{\ell}(0)(\ell=1, \ldots, d)$ :

$$
\begin{aligned}
\mathbb{K}^{n=} & \mathbb{I}^{s_{1}}+\cdots+\mathbb{I}^{s_{m}}, \quad \operatorname{dim} \mathbb{I}^{s_{j}}=s_{j}, j=1, \ldots, m, \\
& s_{1}+\cdots+s_{m}=n .
\end{aligned}
$$

The matrices $A^{1}, \ldots, A^{d}$ can be simultaneously brought in an upper triangular form, and we write again $A^{\ell}$ for the matrices,

$$
A^{\ell}=\left(\begin{array}{cccc}
A_{1}^{\ell} & 0_{s_{1} \times s_{2}} & \ldots & 0_{s_{1} \times s_{m}} \\
0_{s_{2} \times s_{1}} & A_{2}^{\ell} & \ldots & 0_{s_{2} \times s_{m}} \\
\vdots & \vdots & \vdots & \vdots \\
0_{s_{m} \times s_{1}} & 0_{s_{m} \times s_{2}} & \ldots & A_{m}^{\ell}
\end{array}\right), \quad \ell=1, \ldots, d .
$$

If $\mathbb{K}=\mathbb{C}$, the matrix $A_{j}^{\ell}$ is given by

$$
A_{j}^{\ell}=\left(\begin{array}{cccc}
\lambda_{j}^{\ell} & A_{j, 12}^{\ell} & \ldots & A_{j, 1 s_{j}}^{\ell} \\
0 & \lambda_{j}^{\ell} & \ldots & A_{j, 2 s_{j}}^{\ell} \\
\vdots & \vdots & \vdots & \vdots \\
0 & 0 & \ldots & \lambda_{j}^{\ell}
\end{array}\right), \quad \ell=1, \ldots, d, j=1, \ldots, m,
$$

with $\lambda_{j}^{\ell}, A_{j, \nu \mu}^{\ell} \in \mathbb{C}$. On the other hand, if $\mathbb{K}=\mathbb{R}$, then we have, for every $1 \leq j \leq m$ two possibilities: firstly, all $A_{j}^{\ell}(\ell=1, \ldots, d)$ are given by $(1.7)$ with $\lambda_{j}^{\ell} \in \mathbb{R}$. Secondly, $s_{j}=2 \tilde{s_{j}}$ is even and $A_{j}^{\ell}$ is a $\tilde{s_{j}} \times \tilde{s_{j}}$ square block matrix given by

$$
A_{j}^{\ell}=\left(\begin{array}{cccc}
R_{2}\left(\lambda_{j}^{\ell}, \mu_{j}^{\ell}\right) & A_{\ell, j}^{12} & \ldots & A_{\ell j}^{1 \tilde{s_{j}}} \\
0 & R_{2}\left(\lambda_{j}^{\ell}, \mu_{j}^{\ell}\right) & \ldots & A_{\ell j}^{2 s_{j}} \\
\vdots & \vdots & \vdots & \vdots \\
0 & 0 & \ldots & R_{2}\left(\lambda_{j}^{\ell}, \mu_{j}^{\ell}\right)
\end{array}\right), \ell=1, \ldots, d
$$

where

$$
R_{2}(\lambda, \mu):=\left(\begin{array}{cc}
\lambda & \mu \\
-\mu & \lambda
\end{array}\right), \quad \lambda, \mu \in \mathbb{R}
$$

and $A_{\ell j}^{r s}$ are appropriate real matrices. 
Following the decomposition (1.7) (respectively, (1.8)) we define $\tilde{\lambda}^{j}$ by

$$
\tilde{\lambda^{k}}=\left(\lambda_{1}^{k}, \ldots, \lambda_{m}^{k}\right) \in \mathbb{K}^{m}, \quad k=1, \ldots, d .
$$

Then we assume

$$
\tilde{\lambda}^{1}, \cdots, \tilde{\lambda}^{d} \text { are linearly independent in } \mathbb{K}^{m} .
$$

One can easily see that (1.11) is invariantly defined.

We introduce a cone condition. By (1.6) we define

$$
\overrightarrow{\lambda_{j}}={ }^{t}\left(\lambda_{j}^{1}, \cdots, \lambda_{j}^{d}\right) \in \mathbb{K}^{d}, \quad j=1, \ldots, m,
$$

and

$$
\Lambda_{m}:=\left\{\overrightarrow{\lambda_{1}}, \ldots, \overrightarrow{\lambda_{m}}\right\} .
$$

We define the cone $\Gamma\left[\Lambda_{m}\right]$ by

$$
\Gamma\left[\Lambda_{m}\right]=\left\{\sum_{j=1}^{m} t_{j} \overrightarrow{\lambda_{j}} \in \mathbb{K}^{d} ; t_{j} \geq 0, j=1, \ldots, m, \sum_{j=1}^{m} t_{j}^{2} \neq 0\right\} .
$$

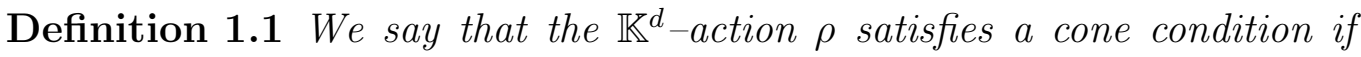
there exists a base $\Lambda_{m} \subset \mathbb{K}^{m}$ such that $\Gamma\left[\Lambda_{m}\right]$ is a proper cone in $\mathbb{K}^{m}$, namely it does not contain a straight real line. If the condition is not satisfied, then, we say that the $\mathbb{K}^{d}$ action is in a Siegel domain.

Note that the definition is invariant under the choice of the basis $\Lambda_{m}$.

Next, we introduce the notion of simultaneous resonances. For $\alpha=$ $\left(\alpha_{1}, \ldots, \alpha_{m}\right) \in \mathbb{K}^{m}, \beta=\left(\beta_{1}, \ldots, \beta_{m}\right) \in \mathbb{K}^{m}$, we set $\langle\alpha, \beta\rangle=\sum_{\nu=1}^{m} \alpha_{\nu} \beta_{\nu}$. For a positive integer $k$ we define $\mathbb{Z}_{+}^{m}(k)=\left\{\alpha \in \mathbb{Z}_{+}^{m} ;|\alpha| \geq k\right\}$. Put

$$
\begin{aligned}
\omega_{j}(\alpha) & =\sum_{\nu=1}^{d}\left|\left\langle\tilde{\lambda}^{\nu}, \alpha\right\rangle-\lambda_{j}^{\nu}\right|, \quad j=1, \ldots, m, \\
\omega(\alpha) & =\min \left\{\omega_{1}(\alpha), \ldots, \omega_{m}(\alpha)\right\} .
\end{aligned}
$$

Definition 1.2 We say that $\Lambda_{m}$ is simultaneously nonresonant (or, in short $\rho$ is simultaneously nonresonant), if

$$
\omega(\alpha) \neq 0, \quad \forall \alpha \in \mathbb{Z}_{+}^{m}(2) .
$$

If (1.17) does not hold, then we say that $\Lambda_{m}$ is simultaneously resonant. 
Clearly, the simultaneously nonresonant condition (1.17) is invariant under the change of the basis $\Lambda_{m}$. We state the first main result of our paper

Theorem 1.3 Let $\rho$ be a $\mathbb{K}^{d}$ analytic action which is simultaneously nonresonant and satisfies the cone condition. Then $\rho$ is linearizable by an analytic change of the variables.

Example 1.4. We compare our theorem with the results of Stolovitch [28] and Zung [32]. Let $\rho$ be a $\mathbb{R}^{2}$ action in $\mathbb{R}^{n}, n \geq 4$ with $m=3$. We choose a basis $\Lambda_{2}$ of $\mathbb{R}^{3}$ such that

$$
\Lambda_{2}=\left\{{ }^{t}(1,1, \nu),{ }^{t}(0,1, \mu)\right\}, \quad \nu, \mu \in \mathbb{R} .
$$

(cf. [DGY] for similar and more general reductions of commuting vector fields on the torus).

We will characterize the set of $(\nu, \mu) \in \mathbb{R}^{2}$ satisfying the cone condition, and determine the simultaneous resonances. By (1.14), $\Gamma\left[\Lambda_{2}\right]$ is generated by the set of vectors $\{(1,0),(1,1),(\nu, \mu)\}$. Hence the cone condition holds if and only if these vectors generate a proper cone, namely $(\nu, \mu)$ is not in the set $\left\{(\nu, \mu) \in \mathbb{R}^{2} ; \nu \leq \mu \leq 0\right\}$. We note that the interesting case is $\mu<\nu \leq 0$, where every generator in (1.18) is in a Siegel domain. Theorem 1.3 can be applied to such a case. In $\S 3$ we will show that if a cone condition is violated, i.e., $\nu<\mu<0$, the linearized overdetermined system of two homological equations has a divergent solution.

Next we will determine $(\nu, \mu)$ so that a simultaneous resonance exists. If $\eta=\left(\eta_{1}, \eta_{2}, \eta_{3}\right) \in \mathbb{Z}_{+}^{3}(2)$ is a simultaneous resonance, we have the following set of equations:

$$
\begin{aligned}
& \text { (1) } \quad \eta_{1}+\eta_{2}+\nu \eta_{3}=1, \eta_{2}+\mu \eta_{3}=0, \\
& \text { (2) } \quad \eta_{1}+\eta_{2}+\nu \eta_{3}=1, \eta_{2}+\mu \eta_{3}=1, \\
& \text { (3) } \quad \eta_{1}+\eta_{2}+\nu \eta_{3}=\nu, \eta_{2}+\mu \eta_{3}=\mu .
\end{aligned}
$$

By elementary computations, in order that one of these equations has a solution $\eta$ the $(\nu, \mu)$ satisfies the following:

a) Case $\nu \leq \mu \leq 0$. The resonance exists iff $(\nu, \mu) \in \mathbb{Q}_{-} \times \mathbb{Q}_{-}$, where $\mathbb{Q}_{-}$is the set of nonpositive rational numbers. The resonance is given by $(1+(\mu-\nu) k,-\mu k, k)$ and $((\mu-\nu) k, 1-k \mu, k)$ where $k \geq 1 /(1-\nu), k \in \mathbb{Z}_{+}$, and $((\nu-\mu)(1-k), \mu(1-k), k)$, where $k \geq(2-\nu)(1-\nu), k \in \mathbb{Z}_{+}$.

b) Case $\nu>\mu$ and $\mu \leq 0$. The resonance is given by $(0,-\mu /(\nu-\mu), 1 /(\nu-\mu))$, 
where $-\mu /(\nu-\mu) \in \mathbb{Z}_{+}, 1 /(\nu-\mu) \in \mathbb{Z}_{+}$and $2 \nu-\mu \leq 1$.

c) Case $\mu>0, \nu \leq \mu$. The resonance is given by $(0,0,1 / \nu)$, when $\nu=\mu$, $\nu \leq 1 / 2, \nu^{-1} \in \mathbb{Z}_{+},(0, \nu, 0)$, when $\nu=\mu \geq 2, \nu \in \mathbb{Z}_{+},((\mu-\nu) / \mu, 0,1 / \mu)$, if otherwise, where $(\mu-\nu) / \mu \in \mathbb{Z}_{+}, 1 / \mu \in \mathbb{Z}_{+}$and $\nu+\mu \leq 1$.

d) Case $\nu>\mu, \mu \geq 0$. The resonance is given by $(\nu-\mu, \mu, 0)$, where $\nu-\mu \in \mathbb{Z}_{+}, \mu \in \mathbb{Z}_{+}$and $\nu \geq 2$.

Let $\nu$ be a negative rational number, $\nu=-k_{1} / k_{2}, k_{1}, k_{2} \in \mathbb{Z}_{+}, k_{2} \neq 0$. Let $\mu$ be a rational number and satisfy $\mu<\nu$. Assume that the nonlinear part of $X^{2}$ is zero. If the nonlinear part of $X^{1}$ consists of the resonant terms of $X^{2}$, then we have $\left[X^{1}, X^{2}\right]=0$. We can easily see that the linearizability of $X^{1}$ holds provided $\mu \neq \nu-1 / k_{2}=-\left(k_{1}+1\right) / k_{2}$.

\section{A cone condition}

We start by showing equivalent forms of the cone condition.

Proposition 2.1 The cone condition is equivalent to each of the following conditions

i) there exist a positive constant $C$ and an integer $k_{0}$ such that

$$
\sum_{k=1}^{d}\left|\sum_{j=1}^{m} \lambda_{j}^{k} \alpha_{j}\right| \geq C_{1}|\alpha|, \quad \forall \alpha \in \mathbb{Z}_{+}^{m}\left(k_{0}\right) .
$$

ii) there exists a nonzero vector $c=\left(c_{1}, \ldots, c_{d}\right) \in \mathbb{C}^{d}$ if $\mathbb{K}=\mathbb{C}$ (respectively, $c=\left(c_{1}, \ldots, c_{d}\right) \in \mathbb{R}^{d}$ if $\left.\mathbb{K}=\mathbb{R}\right)$ such that

$$
c_{1} \tilde{\lambda}^{1}+\cdots+c_{d} \tilde{\lambda}^{d} \text { is in a Poincaré domain, }
$$

namely, the convex hull of the set $\left\{\sum_{j=1}^{d} c_{j} \lambda_{k}^{j} ; k=1, \ldots, m\right\}$ in $\mathbb{C}$ does not contain $0 \in \mathbb{C}$ (respectively,

$$
\text { the real parts of } c_{1} \lambda_{j}^{1}+\cdots+c_{d} \lambda_{j}^{d}, j=1, \ldots, m \text {, are positive.) }
$$

Proof. First we show (2.1). Suppose that (2.1) does not hold. Then there exists a sequence $\alpha^{\ell} \in \mathbb{Z}_{+}^{m}, \ell \in \mathbb{N}$ such that $\left|\alpha^{\ell}\right| \rightarrow \infty(\ell \rightarrow \infty)$ and

$$
\sum_{k=1}^{d}\left|\sum_{j=1}^{m} \lambda_{j}^{k} \alpha_{j}^{\ell}\right| \leq \frac{\left|\alpha^{\ell}\right|}{\ell}, \quad \ell \in \mathbb{N} .
$$


By taking a subsequence, if necessary, we may assume that $\alpha^{\ell} /\left|\alpha^{\ell}\right| \rightarrow t^{0}=$ $\left(t_{1}^{0}, \ldots, t_{m}^{0}\right) \in S_{\ell^{1}}^{1} \bigcap \mathbb{R}_{+}^{m}$ when $\ell \rightarrow \infty$, where $S_{\ell^{1}}^{1}:=\left\{x \in \mathbb{K}^{m} ;\|x\|_{\ell^{1}}=\right.$ $\left.\sum_{j=1}^{m}\left|x_{j}\right|=1\right\}$ stands for the $\ell^{1}$ unit sphere. By letting $\ell \rightarrow \infty$ in (2.4) we get

$$
\sum_{k=1}^{d}\left|\sum_{j=1}^{m} \lambda_{j}^{k} t_{j}^{0}\right|=0
$$

It follows that $\sum_{j=1}^{m} t_{j}^{0} \vec{\lambda}_{j}=0$. Let $J \subset\{1, \ldots, m\}$ be such that $\sum_{j \in J} t_{j}^{0} \vec{\lambda}_{j} \neq$ 0 . Such a set $J$ exists by (1.11). It follows that

$$
0 \neq \sum_{j \in J} t_{j}^{0} \vec{\lambda}_{j}=-\sum_{j \in\{1, \ldots, m\} \backslash J} t_{j}^{0} \vec{\lambda}_{j} .
$$

Hence $\Gamma\left[\Lambda_{m}\right]$ contains a straight line generated by $\sum_{j \in J} t_{j}^{0} \vec{\lambda}_{j} \neq 0$. This contradicts the assumption that $\Gamma\left[\Lambda_{m}\right]$ is a proper cone.

Conversely, suppose that $(2.1)$ is satisfied. We shall show that $\Gamma\left[\Lambda_{m}\right]$ is proper. Indeed, if otherwise, we can find $t^{0}=\left(t_{1}^{0}, \ldots, t_{m}^{0}\right) \in S_{\ell^{1}}^{1} \cap \mathbb{R}_{+}^{m} \backslash 0$ such that

$$
\sum_{j=1}^{m} t_{j}^{0} \lambda_{j}^{k}=0, \quad k=1, \ldots, d .
$$

Because the set $\left\{\alpha /|\alpha| ; \alpha \in \mathbb{Z}_{+}^{m}(2)\right\}$ is dense in $S_{\ell^{1}}^{1} \bigcap \mathbb{R}_{+}^{m}$, there exists a sequence $\alpha^{\ell} \in \mathbb{Z}_{+}^{m}, \ell \in \mathbb{N}$ such that $\left|\alpha^{\ell}\right| \rightarrow \infty(\ell \rightarrow \infty)$ and $\lim _{\ell \rightarrow \infty} \alpha^{\ell} /\left|\alpha^{\ell}\right|=$ $t^{0}$. Therefore, in view of $(2.5)$, we get

$$
\lim _{\ell \rightarrow \infty}\left(\frac{1}{\left|\alpha^{\ell}\right|} \sum_{k=1}^{d}\left|\sum_{j=1}^{m} \lambda_{j}^{k} \alpha_{j}^{\ell}\right|\right)=0
$$

which contradicts (2.1)

Next, we show ii). Suppose that $\Gamma\left[\Lambda_{m}\right]$ be a proper cone in $\mathbb{K}^{d}$. Then we can find $c=\left(c_{1}, \ldots, c_{d}\right) \in \mathbb{C}^{d}$ such that $\Gamma\left[\Lambda_{m}\right]$ is contained in the real half-space $P_{c}:=\left\{z \in \mathbb{K}^{d}, \operatorname{Re}\left(\sum_{k=1}^{d} c_{k} z_{k}\right)>0\right\}$. Therefore

$$
0<\operatorname{Re}\left(\sum_{k=1}^{d} c_{k} \sum_{j=1}^{m} t_{j} \lambda_{j}^{k}\right)=\sum_{j=1}^{m} t_{j} \operatorname{Re}\left(\sum_{k=1}^{d} c_{k} \lambda_{j}^{k}\right)
$$


for all $t \in \mathbb{R}_{+}^{m} \backslash 0$, which yields $\operatorname{Re}\left(\sum_{k=1}^{d} c_{k} \lambda_{j}^{k}\right)>0$ for $j=1, \ldots, m$. We note that, if $\mathbb{K}=\mathbb{R}$, then the use of the real part in the definition of the half-space is superfluous. Finally, we readily see, from (2.2) that, if $\mathbb{K}=\mathbb{C}$ (respectively, $(2.3)$ if $\mathbb{K}=\mathbb{R}$ ), then the cone $\Gamma\left[\Lambda_{m}\right]$ is contained in $P_{c}$. Hence $\Gamma\left[\Lambda_{m}\right]$ is proper. The proof is complete.

The proof of Theorem 1.3 follows from

Proposition 2.2 Let the action $\rho$ satisfy the cone condition. Then we can find a vector field in the corresponding Lie algebra which is nonresonant and is in the Poincaré domain.

Proof. By ii) of Proposition 2.1 we can find a Poincaré vector field in the Lie algebra as a linear combination of a base corresponding to (2.2). Let $c_{\nu}$ be the number in (2.2), and define $\tilde{\lambda}^{0}:=\left(\lambda_{1}^{0}, \ldots, \lambda_{m}^{0}\right)=\sum_{\nu=1}^{d} c_{\nu} \tilde{\lambda}^{\nu}$. Consider

$$
\left\langle\tilde{\lambda}^{0}, \alpha\right\rangle-\lambda_{j}^{0}=\sum_{\nu=1}^{d} c_{\nu}\left(\left\langle\tilde{\lambda}^{\nu}, \alpha\right\rangle-\lambda_{j}^{\nu}\right) .
$$

Because $\sum_{\nu=1}^{d}\left|\left\langle\tilde{\lambda}^{\nu}, \alpha\right\rangle-\lambda_{j}^{\nu}\right| \neq 0$ for $\forall \alpha \in \mathbb{Z}_{+}^{m}(2)$ by the simultaneous nonresonant condition it follows that the set $\left\langle\tilde{\lambda}^{0}, \alpha\right\rangle-\lambda_{j}^{0}=0$ in $c=\left(c_{1}, \ldots, c_{d}\right) \in \mathbb{C}^{d}$ is a hyperplane. It follows that the set

$$
\left\{c=\left(c_{1}, \ldots, c_{d}\right) \in \mathbb{C}^{d} ;\left\langle\tilde{\lambda}^{0}, \alpha\right\rangle-\lambda_{j}^{0}=0, \exists j, 1 \leq j \leq m, \exists \alpha \in \mathbb{Z}_{+}^{m}(2)\right\}
$$

is a countable union of nowhere dense closed set. Therefore we can find $c=\left(c_{1}, \ldots, c_{d}\right)$ for which $\sum_{\nu=1}^{d} c_{\nu} \tilde{\lambda}^{\nu}$ satisfies a nonresonant condition and the cone condition. This proves Proposition 2.2.

We propose a geometric expression of a cone condition

Definition 2.3 Let $r>0$ and $g$ be a Riemannian metric on $\mathbb{R}^{n}$. We denote by $\langle\cdot, \cdot\rangle_{g}$ and $\|\cdot\|_{g}$ the inner product and the norm with respect to $g$, respectively. We say that $\mathcal{X}_{\nu}:=\sum_{j=1}^{n} X_{j}^{\nu}(x) \partial_{x_{j}}(\nu=1, \ldots, d)$ are simultaneously transversal to the sphere $\|x\|_{g}=r$ if, the vectors $X^{\nu}:=\left(X_{1}^{\nu}, \ldots, X_{n}^{\nu}\right)$ $(\nu=1, \ldots, d)$ satisfy

$$
\sum_{\nu=1}^{d}\left|\left\langle X^{\nu}, x\right\rangle_{g}\right| \neq 0, \quad \forall x, \quad\|x\|_{g}=r .
$$


Theorem 2.4 Let $r>0$. Suppose that $\mathcal{B}_{\nu}:=\sum_{j=1}^{n}\left(x A^{\nu}\right)_{j} \partial_{x_{j}}(\nu=1, \ldots, d)$ be a commuting system of semi-simple linear real vector fields in $\mathbb{R}^{n}$. We choose a real nonsingular matrix $P$ such that $\Lambda^{\nu}=P^{-1} A^{\nu} P$ is a block diagonal matrix given by $\Lambda^{\nu}=\operatorname{diag}\left\{R_{2}\left(\xi_{1}^{\nu}, \eta_{1}^{\nu}\right), \ldots, R_{2}\left(\xi_{n_{1}}^{\nu}, \eta_{n_{1}}^{\nu}\right), \lambda_{n_{1}+1}^{\nu}, \ldots, \lambda_{n}^{\nu}\right\}$ for some integer $n_{1} \leq n$.. Let $g$ be a Riemannian metric defined by $P^{t} P$. Then the following conditions are equivalent.

(a) $\mathcal{B}_{\nu}(\nu=1, \ldots, d)$ are simultaneously transversal to the sphere $\|x\|_{g}=r$.

(b) $\mathcal{B}_{\nu}(\nu=1, \ldots, d)$ satisfy a cone condition.

(c) There exist real numbers $c_{\nu}(\nu=1, \ldots, d)$ such that $\sum_{\nu=1}^{d} c_{\nu} \mathcal{B}_{\nu}$ is transversal to the sphere $\|x\|_{g}=r$.

Proof. We note that $\langle x, y\rangle_{g}=\langle P x, P y\rangle$ and $\|x\|_{g}=\|P x\|$. By inserting the relation $A^{\nu}=P \Lambda^{\nu} P^{-1}$ into (2.7) we can easily see that the simultaneous transversality condition is equivalent to

$$
\sum_{\nu=1}^{d}\left|\left\langle y \Lambda^{\nu}, y\right\rangle\right| \neq 0, \quad \forall y=\left(y_{1}, \ldots, y_{n}\right), \quad\|y\|=1 .
$$

By definition, (2.8) can be written in

$$
\sum_{\nu=1}^{d}\left|\sum_{j=1}^{n_{1}} \xi_{j}^{\nu}\left(y_{2 j-1}^{2}+y_{2 j}^{2}\right)+\sum_{j=n_{1}+1}^{n} y_{j}^{2} \lambda_{j}^{\nu}\right| \neq 0, \quad \forall y, \quad\|y\|=1 .
$$

We define $t=\left(t_{1}, \ldots, t_{n}\right), t \in \mathbb{R}_{+}^{n},|t|=1$ by $t_{j}=\left(y_{2 j-1}^{2}+y_{2 j}^{2}\right) / 2$ if $j \leq n_{1}$ and $t_{j}=y_{j}^{2}$ if $j>2 n$. Noting that $\xi^{n} u_{j}\left(y_{2 j-1}^{2}+y_{2 j}^{2}\right)=2 t_{j} \xi_{j}^{\nu}=t_{j}\left(\xi_{j}^{\nu}+i \eta_{j}^{\nu}+\xi_{j}^{\nu}-i \eta_{j}^{\nu}\right)$ we see that (2.9) is written in $\sum_{\nu=1}^{d}\left|\sum_{j=1}^{n} t_{j} \lambda_{j}^{\nu}\right| \neq 0$ for every $t \in \mathbb{R}_{+}^{n}$ and $|t|=1$. This is equivalent to the cone condition by definition. Hence we have proved the equivalence of (a) and (b).

By Proposition 2.1 the condition (b) is equivalent to the existence of real numbers $c_{\nu}(\nu=1, \ldots, d)$ such that $\sum_{\nu=1}^{d} c_{\nu} \mathcal{B}_{\nu}$ is a Poincaré vector field. By what we have proved in the above $(d=1)$ this is equivalent to say that $\sum_{\nu=1}^{d} c_{\nu} \mathcal{B}_{\nu}$ is transversal to the sphere $\|x\|_{g}=r$. Hence we have proved Theorem 2.3. 


\section{Divergent solutions of overdetermined sys- tems of linearized homological equations}

We now study the action $\rho_{\text {lin }}$ which is in a Siegel domain and admits a Jordan block. We assume that the action is formally (simultaneously) linearizable and that does not satisfy the cone condition. We shall show that the unique formal solution of a linearized homological equation diverges.

Let $\mathbb{C}_{2}^{n}\{x\}$ be the set of $n$ vector functions of convergent power series of $x$ without constant and linear terms. We examine the system of the linearized homology equation

$$
L_{A} v=\left(L_{1} v, \ldots, L_{d} v\right)=f, \quad f:=\left(f_{1}, \ldots, f_{d}\right) \in\left(\mathbb{C}_{2}^{n}\{x\}\right)^{d},
$$

where

$$
L_{j} v=\left\langle A_{j} x, \partial_{x}\right\rangle v-A_{j} v, \quad j=1, \ldots, d,
$$

under the compatibility conditions

$$
L_{j} f_{k}=L_{k} f_{j}, \quad j, k=1, \ldots, d .
$$

First we consider a 2- $\mathbb{C}$ action studied in Example 1.4. We assume that there exists a vector field in the two-dimensional Lie algebra which is not semisimple. In view of Example 1.4 we can choose a base $X_{1}, X_{2}$ with linear parts $A_{j} \in G L(4 ; \mathbb{C})$ satisfying $\operatorname{spec}\left(A_{1}\right)=\{1,1, \nu, \nu\}$ and $\operatorname{spec}\left(A_{2}\right)=$ $\{0,1, \mu, \mu\}$, respectively, where $\nu \leq \mu \leq 0,(\nu, \mu) \notin \mathbb{Q} \times \mathbb{Q}$, and

$$
A_{1}=\left(\begin{array}{cccc}
1 & 0 & 0 & 0 \\
0 & 1 & 0 & 0 \\
0 & 0 & \nu & \varepsilon \\
0 & 0 & 0 & \nu
\end{array}\right), \quad A_{2}=\left(\begin{array}{cccc}
0 & 0 & 0 & 0 \\
0 & 1 & 0 & 0 \\
0 & 0 & \mu & \varepsilon_{0} \varepsilon \\
0 & 0 & 0 & \mu
\end{array}\right)
$$

where $\varepsilon \neq 0$ and $\varepsilon_{0} \in \mathbb{C}$. We can make $|\varepsilon|>0$ arbitrarily small by an appropriate linear change of variables.

Let $\omega(\alpha)$ be defined by (1.16). We say that the simultaneous Diophantine

order of $\left\{\operatorname{spec}\left(A_{1}\right), \operatorname{spec}\left(A_{2}\right)\right\}$ is $\tau_{0}$, if, for every $\tau>\tau_{0}$ there exists $C=$ $C_{\tau}>0$ such that

$$
\omega(\alpha) \geq C|\alpha|^{-\tau}, \quad \forall \alpha \in \mathbb{Z}_{+}^{4}(2),
$$


while, for every $\tau<\tau_{0}$ there exists a subsequence $\alpha_{\ell} \in \mathbb{Z}_{+}^{4}(2)(\ell=1,2, \ldots)$ such that

$$
\omega(\alpha) \leq\left|\alpha_{\ell}\right|^{-\tau}, \quad \ell \in \mathbb{N} .
$$

By a classical result in the number theory we have $\tau_{0} \geq 1 / 2$ ( cf. [26] or [22] ). We say that $\alpha$ and $\beta$ are simultaneously Liouville, if (3.5) holds for every $\tau>0$.

Let $\sigma \geq 1$. We say that a formal power series $f(x)=\sum_{\alpha} f_{\alpha} x^{\alpha}$ is in a Gevrey space $G_{2}^{\sigma}\left(\mathbb{C}^{4}\right)$ if $f_{\alpha}=0$ for $|\alpha| \leq 1$ and, there exist $C>0$ and $R>0$ such that

$$
\left|f_{\alpha}\right| \leq C R^{|\alpha|}|\alpha|^{\sigma}, \quad \forall \alpha \in \mathbb{Z}_{+}^{4} .
$$

We consider the following equation

$$
L_{A} v:=\left(L_{1} v, L_{2} v\right)=f, \quad f=\left(f_{1}, f_{2}\right) \in\left(\mathbb{C}_{2}^{4}\{x\}\right)^{2}, x \in \mathbb{C}^{4},
$$

where $\left(f_{1}, f_{2}\right)$ satisfies the compatibility condition $L_{1} f_{2}=L_{2} f_{1}$. Then we have

Theorem 3.1 Assume that $\nu<\mu \leq 0$ and $\varepsilon_{0} \neq 0$. Then there exists $f=\left(f_{1}, f_{2}\right) \in\left(\mathbb{C}_{2}^{4}\{x\}\right)^{2}$ such that $L_{1} f_{2}=L_{2} f_{1}$ and the equation (3.6) has a formal power series solution $v \notin \bigcup_{1 \leq \sigma<5 / 2} G_{2}^{\sigma}\left(\mathbb{C}^{4}\right)$.

Furthermore, suppose that $(\nu, \mu) \notin \mathbb{Q} \times \mathbb{Q}$, (3.4), (3.5) and $\tau_{0}<+\infty$ hold. Then (3.6) has a unique solution $v \in \bigcap_{\sigma>3+2 \tau_{0}} G_{2}^{\sigma}\left(\mathbb{C}^{4}\right)$ for every $\left(f_{1}, f_{2}\right) \in$ $\left(\mathbb{C}_{2}^{4}\{x\}\right)^{2}$ satisfying $L_{1} f_{2}=L_{2} f_{1}$.

The proof of this theorem follows from the following propositions.

Proposition 3.2 Suppose that $\nu<\mu \leq 0, \varepsilon_{0} \neq 0$, and $(\nu, \mu) \notin \mathbb{Q} \times \mathbb{Q}$. Then there exist real numbers $c_{1}$ and $c_{2}$ such that for any $g \in \mathbb{C}_{2}^{4}\{x\}$ there exist $f_{j} \in \mathbb{C}_{2}^{4}\{x\}(j=1,2)$ such that $L_{1} f_{2}=L_{2} f_{1}$ and $g=\sum_{j=1}^{2} c_{j} f_{j}$. Moreover, $B:=c_{1} A_{1}+c_{2} A_{2}$ is nonresonant, and $\omega$ defined by (1.16) for $\operatorname{spec}(B)$ satisfies (3.5).

Proposition 3.3 Suppose that $\nu<\mu \leq 0, \varepsilon_{0} \neq 0$ and $(\nu, \mu) \notin \mathbb{Q} \times \mathbb{Q}$. Let $c_{1}$ and $c_{2}$ be the numbers given by Proposition 3.2. Then there exists $g \in \mathbb{C}_{2}^{4}\{x\}$ such that the homology equation $L_{B} v=g$ with $B=c_{1} A_{1}+c_{2} A_{2}$ has a unique formal power series solution $v$ which is not contained in $\bigcup_{1 \leq \sigma<5 / 2} G_{2}^{\sigma}\left(\mathbb{C}^{4}\right)$. 
Remark 3.4 Our divergence results imply in the case of a single holomorphic vector field, that generically vector fields obtained by nonlinear holomorphic perturbations are nonlinearizable (see R. Pérez Marco [25] and [20] for more details). As to the case of smooth $C^{\infty}$ hyperbolic $\mathbb{R}^{2}$ actions we refer [10]. We point out that the divergence in Gevrey classes of formal solutions of overdetermined systems of linear homological equations generalize those for the single vector fields in the presence of nontrivial Jordan blocks (see [16], [18]).

First we will show Theorem 3.1, assuming Propositions 3.2 and 3.3.

Proof of Theorem 3.1. We will prove the former half. By the result of Example 1.4 of the case (a), we know that if $(\nu, \mu) \in \mathbb{Q} \times \mathbb{Q}$, then (3.6) has an infinite resonance. It follows that (3.6) with $f=0$ has a formal power series solution $v \notin \bigcup_{1 \leq \sigma<5 / 2} G_{2}^{\sigma}\left(\mathbb{C}^{4}\right)$, because (3.6) is a linear equation. Next we assume $(\nu, \mu) \notin \mathbb{Q} \times \mathbb{Q}$. By Proposition 3.3 we can choose $g \in \mathbb{C}_{2}^{4}\{x\}$ such that the unique solution $v$ of $L_{B} v=g$ with $B=c_{1} A_{1}+c_{2} A_{2}$ is not contained in $\bigcup_{1 \leq \sigma<5 / 2} G_{2}^{\sigma}\left(\mathbb{C}^{4}\right)$. By Proposition 3.2 we choose $f_{j} \in \mathbb{C}_{2}^{4}\{x\}$ $(j=1,2)$ such that $L_{1} f_{2}=L_{2} f_{1}$ and $g=\sum_{j=1}^{2} c_{j} f_{j}$. Because the solution $v$ of the system of equations $L_{A} v=f$ is a unique solution of a single equation $L_{B} v=g$ we see that $v$ is not contained in $\bigcup_{1 \leq \sigma<5 / 2} G_{2}^{\sigma}\left(\mathbb{C}^{4}\right)$. This proves the former half of the theorem.

We will prove the latter half. We consider the system of equations $L_{j} v=f_{j}(j=1,2)$, where $L_{1} f_{2}=L_{2} f_{1}$. For the sake of simplicity, we assume that $B$ is put in a Jordan normal form with the diagonal part $B^{0}:=\operatorname{diag}\left\{\lambda_{1}, \lambda_{2}, \lambda_{3}, \lambda_{3}\right\}$. The off diagonal element of $B$ is denoted by $\varepsilon_{1}$. The homology operator corresponding to $B$ is given by

$$
\begin{aligned}
L_{B} v & =\left\langle B^{0} x, \partial_{x}\right\rangle v+\varepsilon_{1} R[v]-B v, \quad v \in \mathbb{C}_{2}^{4}\{x\}, \\
\left\langle B^{0} x, \partial_{x}\right\rangle v & =\sum_{|\alpha| \geq 2}\left(\lambda_{1} \alpha_{1}+\lambda_{2} \alpha_{2}+\lambda_{3}\left(\alpha_{3}+\alpha_{4}\right)\right) v_{\alpha} x^{\alpha},
\end{aligned}
$$

where $v(x)=\sum_{|\alpha| \geq 2} v_{\alpha} x^{\alpha}$ and

$$
R[v]=\sum_{|\alpha| \geq 2}\left(\alpha_{3}+1\right) v_{\left(\alpha_{1}, \alpha_{2}, \alpha_{3}+1, \alpha_{4}-1\right)} x^{\alpha} .
$$

For $g(x)=\left(g_{1}, g_{2}, g_{3}, g_{4}\right)^{t} \in \mathbb{C}_{2}^{4}\{x\}$ we expand $g_{k}(x)$ in the Taylor series $g_{k}(x)=\sum_{\alpha} g_{\alpha ; k} x^{\alpha}$. For nonnegative integers $N, \alpha_{1}$ and $\alpha_{2}$ we define $V_{k}$ and 
$G_{k}$ by

$$
V_{k}:=\left\{v_{\left(\alpha_{1}, \alpha_{2}, N-\ell, \ell\right) ; k}\right\}_{\ell=0}^{N}, \quad G_{k}:=\left\{g_{\left(\alpha_{1}, \alpha_{2}, N-\ell, \ell\right) ; k}\right\}_{\ell=0}^{N} \quad(k=1,2,3,4) .
$$

In view of (3.9), the equation $L_{B} v=g$ is equivalent to

$$
\begin{aligned}
\left(\lambda_{1} \alpha_{1}+\lambda_{2} \alpha_{2}+\lambda_{3} N-\lambda_{1}\right) V_{1}+\varepsilon_{1} \mathcal{M}_{N} V_{1} & =G_{1}, \\
\left(\lambda_{1} \alpha_{1}+\lambda_{2} \alpha_{2}+\lambda_{3} N-\lambda_{2}\right) V_{2}+\varepsilon_{1} \mathcal{M}_{N} V_{2} & =G_{2}, \\
\left(\lambda_{1} \alpha_{1}+\lambda_{2} \alpha_{2}+\lambda_{3}(N-1)\right) V_{3}+\varepsilon_{1} \mathcal{M}_{N} V_{3} & =G_{3}+\varepsilon_{1} V_{4}, \\
\left(\lambda_{1} \alpha_{1}+\lambda_{2} \alpha_{2}+\lambda_{3}(N-1)\right) V_{4}+\varepsilon_{1} \mathcal{M}_{N} V_{4} & =G_{4},
\end{aligned}
$$

where $\mathcal{M}_{N}$ is given by

$$
\mathcal{M}_{N}=\left(\begin{array}{ccccccc}
0 & 0 & 0 & \ldots & 0 & 0 & 0 \\
N & 0 & 0 & \ldots & 0 & 0 & 0 \\
0 & N-1 & 0 & \ldots & 0 & 0 & 0 \\
0 & 0 & N-2 & \ldots & 0 & 0 & 0 \\
\vdots & \vdots & \vdots & \ddots & \vdots & \vdots & \vdots \\
0 & 0 & 0 & \ldots & 2 & 0 & 0 \\
0 & 0 & 0 & \ldots & 0 & 1 & 0
\end{array}\right), \quad N \geq 1
$$

and $\mathcal{M}_{0}=0$. We note that, for the equation $L_{1} v=f_{1}$ we have $\lambda_{1}=\lambda_{2}=1$, $\lambda_{3}=\nu, \varepsilon_{1}=\varepsilon$, while for $L_{2} v=f_{2}$ we have $\lambda_{1}=0, \lambda_{2}=1, \lambda_{3}=\mu, \varepsilon_{1}=\varepsilon_{0} \varepsilon$.

Let $f^{j}(x)=\left(f_{1}^{j}(x), \ldots, f_{4}^{j}(x)\right)$ and let $f_{k}^{j}(x)=\sum_{\alpha} f_{\alpha ; k}^{j} x^{\alpha}(j=1,2 ; k=$ $1, \ldots, 4)$ be the Taylor expansion of $f_{k}^{j}(x)$. We substitute the expansions of $v$ and $f^{j}$ into the equations $L_{j} v=f^{j}$. For every $\left(\alpha_{1}, \alpha_{2}\right) \in \mathbb{Z}_{+}^{2}$ and $N \in \mathbb{Z}_{+}$ such that $\alpha_{1}+\alpha_{2}+N \geq 2$ we compare the coefficients of $x^{\alpha}\left(\alpha_{3}+\alpha_{4}=N\right)$ with homogeneous degree $\alpha_{1}+\alpha_{2}+N$. If we set

$$
F^{j}=\left(F_{1}^{j}, \ldots, F_{4}^{j}\right), \quad F_{k}^{j}=\left\{f_{\left(\alpha_{1}, \alpha_{2}, N-r, r\right) ; k}^{j}\right\}_{r=0}^{N}, \quad j=1,2 ; k=1, \ldots, 4,
$$

and $V=\left(V_{1}, \ldots, V_{4}\right), V_{k}:=\left\{v_{\left(\alpha_{1}, \alpha_{2}, N-\ell, \ell\right) ; k}\right\}_{\ell=0}^{N}(k=1,2,3,4), H=\left(0,0, V_{4}, 0\right)$, then we can write the system of equations $L_{j} v=f^{j}(j=1,2)$ in the following form

$$
\mathcal{A} V=F^{1}+\varepsilon H, \quad \mathcal{B} V=F^{2}+\varepsilon \varepsilon_{0} H,
$$


where the matrices $\mathcal{A}$ and $\mathcal{B}$ are the block diagonal matrices given by

$$
\begin{gathered}
\mathcal{A}:=\operatorname{diag}\left\{\mathcal{A}_{1}, \mathcal{A}_{2}, \mathcal{A}_{3}, \mathcal{A}_{4}\right\}=\operatorname{diag}\left(\begin{array}{c}
\left.\left(\alpha_{1}+\alpha_{2}+\nu N-1\right) I d+\varepsilon M_{N}\right) \\
\left.\left(\alpha_{1}+\alpha_{2}+\nu N-1\right) I d+\varepsilon M_{N}\right) \\
\left.\left(\alpha_{1}+\alpha_{2}+\nu N-\nu\right) I d+\varepsilon M_{N}\right) \\
\left.\left(\alpha_{1}+\alpha_{2}+\nu N-\nu\right) I d+\varepsilon M_{N}\right)
\end{array}\right), \\
\mathcal{B}:=\operatorname{diag}\left\{\mathcal{B}_{1}, \mathcal{B}_{2}, \mathcal{B}_{3}, \mathcal{B}_{4}\right\}=\operatorname{diag}\left(\begin{array}{c}
\left.\left(\alpha_{2}+\mu N\right) I d+\varepsilon_{0} \varepsilon M_{N}\right) \\
\left.\left(\alpha_{2}+\mu N-1\right) I d+\varepsilon_{0} \varepsilon M_{N}\right) \\
\left.\left(\alpha_{2}+\mu N-\mu\right) I d+\varepsilon_{0} \varepsilon M_{N}\right) \\
\left.\left(\alpha_{2}+\mu N-\mu\right) I d+\varepsilon_{0} \varepsilon M_{N}\right)
\end{array}\right) .
\end{gathered}
$$

We will construct a formal power series solution $V$ from (3.16). Because $(\nu, \mu) \notin \mathbb{Q} \times \mathbb{Q}$ either $\nu$ or $\mu$ is an irrational number. Suppose that $\nu$ is an irrational number. We want to show that for each $k=1, \ldots, 4$ either $\mathcal{A}_{k}$ or $\mathcal{B}_{k}$ is nonsingular. In order to see this, suppose that $|\alpha|=\alpha_{1}+\alpha_{2}+N \geq 2$. If $N \neq 0,1$, then by the irrationality of $\nu$, the matrices $\mathcal{A}_{k}(k=1, \ldots, 4)$ are nonsingular. If $N=0$ or $N=1$, then by the condition $\alpha_{1}+\alpha_{2}+N \geq 2, \mathcal{A}_{k}$ $(k=1, \ldots, 4)$ are nonsingular. Similarly, if $\mu$ is an irrational number, then we can show that either $\mathcal{A}_{k}$ or $\mathcal{B}_{k}$ is nonsingular for each $k=1, \ldots, 4$.

First we will determine $V_{4}$. By inductive arguments and $L_{1} f^{2}=L_{2} f^{1}$ we get

$$
\begin{aligned}
v_{\left(\alpha_{1}, \alpha_{2}, N-\ell, \ell\right) ; 4} & =\sum_{r=0}^{\ell} \frac{\left(-\varepsilon_{1}\right)^{r}}{\left(\lambda_{1} \alpha_{1}+\lambda_{2} \alpha_{2}+\lambda_{3}(N-1)\right)^{r+1}} \\
& \times \frac{(N-\ell+r) !}{(N-\ell) !} g_{\left(\alpha_{1}, \alpha_{2}, N-\ell+r, \ell-r\right) ; 4}
\end{aligned}
$$

for $\ell=0,1, \ldots, N$, provided $\lambda_{1} \alpha_{1}+\lambda_{2} \alpha_{2}+\lambda_{3}(N-1) \neq 0$. Note that, if $\mathcal{A}_{4}$ is nonsingular, then (3.19) is valid for $\lambda_{1}=\lambda_{2}=1, \lambda_{3}=\nu, \varepsilon_{1}=$ $\varepsilon, g_{\left(\alpha_{1}, \alpha_{2}, N-\ell+r, \ell-r\right) ; 4}=f_{\left(\alpha_{1}, \alpha_{2}, N-\ell+r, \ell-r\right) ; 4}^{1}$, while if $\mathcal{B}_{4}$ is nonsingular, then (3.19) is valied for $\lambda_{1}=0, \lambda_{2}=1, \lambda_{3}=\mu, \varepsilon_{1}=\varepsilon_{0} \varepsilon, g_{\left(\alpha_{1}, \alpha_{2}, N-\ell+r, \ell-r\right) ; 4}=$ $f_{\left(\alpha_{1}, \alpha_{2}, N-\ell+r, \ell-r\right) ; 4}^{2}$. Similar explicit formulas are derived for $v_{\alpha_{1}, \alpha_{2}, N-\ell, \ell ; k}, k=$ 1,2 . As to the term $v_{\left(\alpha_{1}, \alpha_{2}, N-\ell, \ell\right) ; 3}$, there appears the term $\varepsilon_{1} V_{4}^{N}$ in the righthand side of (3.12).

By (3.4) we have

$$
\left|\alpha_{1}+\alpha_{2}+\nu N-\nu\right|+\left|\alpha_{2}+\mu N-\mu\right| \geq C\left|\alpha_{1}+\alpha_{2}+N\right|^{-\tau}
$$


for some $C>0$. It follows that either $\left|\alpha_{1}+\alpha_{2}+\nu N-\nu\right| \geq C\left|\alpha_{1}+\alpha_{2}+N\right|^{-\tau} / 2$ or $\left|\alpha_{2}+\mu N-\mu\right| \geq C\left|\alpha_{1}+\alpha_{2}+N\right|^{-\tau} / 2$ holds. Suppose that the former estimate holds. We have the same estimate in case the latter inequality holds. Let $\tau$ be such that $\tau>\tau_{0}$. Then we have

$$
\begin{aligned}
\left|\alpha_{1}+\alpha_{2}+\nu(N-1)\right|^{r+1} & \geq(C / 2)^{r+1}\left|\alpha_{1}+\alpha_{2}+N\right|^{-\tau(r+1)} \\
& \geq\left|\alpha_{1}+\alpha_{2}+N\right|^{-\tau(N+1)} .
\end{aligned}
$$

Noting that $(N-\ell+r) ! /(N-\ell) ! \leq N$ !, we see from (3.19) that if $g_{\left(\alpha_{1}, \alpha_{2}, N-\ell+r, \ell-r\right) ; 4}$ has a $G^{s}$ estimate, then $v_{\left(\alpha_{1}, \alpha_{2}, N-\ell, \ell\right) ; 4}$ has a $G^{s+\tau+1}$ estimate. Especially, $v_{\left(\alpha_{1}, \alpha_{2}, N-\ell, \ell\right) ; 4}$ has the $G^{\tau+2}$ estimate. Similarly, we can easily see that $v_{\left(\alpha_{1}, \alpha_{2}, N-\ell, \ell\right) ; j}(j=1,2,4)$ have the $G^{\tau+2}$ estimate. Next we determine $v_{\left(\alpha_{1}, \alpha_{2}, N-\ell, \ell\right) ; 3}$ by a similar relation like (3.19). We can easily see that there appears $v_{\left(\alpha_{1}, \alpha_{2}, N-\ell, \ell\right) ; 4}$ in the right-hand side of the recurrence relation. Hence the right-hand side has the $G^{\tau+2}$ estimate. It follows that $v_{\left(\alpha_{1}, \alpha_{2}, N-\ell, \ell\right) ; 3}$ has the $G^{2 \tau+3}$ estimate. Since $\tau>\tau_{0}$ is arbitray, $v_{\left(\alpha_{1}, \alpha_{2}, N-\ell, \ell\right) ; 3}$ has the $G^{\sigma}$ estimate for $\sigma>3+2 \tau_{0}$. This ends the proof of Theorem 3.1.

Proof of Proposition 3.2. The eigenvalues of $B:=c_{1} A_{1}+c_{2} A_{2}$ is given by $c_{1}, c_{1}+c_{2}, c_{1} \nu+c_{2} \mu$ with multiplicity. We shall show that there exists a set $E \subset \mathbb{R}^{2}$ with Lebesgue measure zero such that if $\left(c_{1}, c_{2}\right) \notin E$, then $B$ is nonresonant. For every $\alpha=\left(\alpha_{1}, \ldots, \alpha_{4}\right) \in \mathbb{Z}_{+}^{4},|\alpha| \geq 2$, the resonance relations are given by

$$
c_{1} \alpha_{1}+\left(c_{1}+c_{2}\right) \alpha_{2}+\left(c_{1} \nu+c_{2} \mu\right) \alpha_{3}=c_{1},
$$

and the ones with $c_{1}$ in the right-hand side replaced by $c_{1}+c_{2}$ and $c_{1} \nu+c_{2} \mu$, respectively. Because the argument is similar, we consider the first relation. It follows from (3.22) that

$$
c_{1}\left(\alpha_{1}+\alpha_{2}+\nu\left(\alpha_{3}+\alpha_{4}\right)-1\right)+c_{2}\left(\alpha_{2}+\mu\left(\alpha_{3}+\alpha_{4}\right)\right)=0
$$

Because $(\nu, \mu) \notin \mathbb{Q} \times \mathbb{Q}$ and $|\alpha| \geq 2$, we can easily see that either $\alpha_{1}+\alpha_{2}+$ $\nu\left(\alpha_{3}+\alpha_{4}\right)-1 \neq 0$ or $\alpha_{2}+\mu\left(\alpha_{3}+\alpha_{4} \neq 0\right.$ holds. Hence the set of $\left(c_{1}, c_{2}\right) \in \mathbb{R}^{2}$ satisfying (3.22) is a straight line. Therefore the set $E$ of all $\left(c_{1}, c_{2}\right)$ satisfying a resonance relations has Lebesgue measure zero.

In order to see that $\operatorname{spec}(B)$ satisfies $(3.5)$, let $\tilde{\omega}_{j}(\alpha)\left(\alpha \in \mathbb{Z}_{+}^{4}\right)$ be defined by $(1.15)$ for $B$. Then there exists $K>0$ such that $\tilde{\omega}_{j}(\alpha) \leq K \omega_{j}(\alpha)$ for $j=1, \ldots, 4$ and all $\alpha \in \mathbb{Z}_{+}^{4}$. It follows that (3.5) holds for $\tilde{\omega}_{j}(\alpha)$. 
Let $\left(c_{1}, c_{2}\right) \notin E$ and $g \in \mathbb{C}_{2}^{4}$ be given. We want to solve the system of equations

$$
L_{1} f^{2}=L_{2} f^{1}, \quad c_{1} f^{1}+c_{2} f^{2}=g .
$$

By expanding $f^{j}(x)=\left(f_{1}^{j}, \ldots, f_{4}^{j}\right)$ into the Taylor series we define $F^{j}$ by (3.15). We similarly define $G=\left(G_{1}, \ldots, G_{4}\right), G_{k}=\left\{g_{\left(\alpha_{1}, \alpha_{2}, N-r, r\right) ; k}\right\}_{r=0}^{N}$, where $g(x)=\left(g_{1}, \ldots, g_{4}\right), g_{k}(x)=\sum_{\alpha} g_{\alpha ; k} x^{\alpha}$. We set $H^{1}:=\left(0,0, F_{4}^{1}, 0\right)$ and $H^{2}:=\left(0,0, F_{4}^{2}, 0\right)$. We substitute the expansions of $f^{j}$ and $g$ into (3.23). For every $\left(\alpha_{1}, \alpha_{2}\right) \in \mathbb{Z}_{+}^{2}$ and $N \in \mathbb{Z}_{+}$such that $\alpha_{1}+\alpha_{2}+N \geq 2$ we compare the coefficients of $x^{\alpha}$ of homogeneous degree $\alpha_{1}+\alpha_{2}+N$. Then we can write (3.23) in the following form

$$
\mathcal{A} F^{2}-\mathcal{B} F^{1}+\varepsilon H^{2}-\varepsilon \varepsilon_{0} H^{1}=0, \quad c_{1} F^{1}+c_{2} F^{2}=G,
$$

where $\mathcal{A}$ and $\mathcal{B}$ are given by (3.17) and (3.18).

First we will construct a formal power series solution $F^{j}(j=1,2)$ of (3.24) for a given $G$. Because we know that (cf. the proof of Theorem 3.1) either $\mathcal{A}_{k}$ or $\mathcal{B}_{k}$ is nonsingular for each $k=1, \ldots, 4$, it follows from $(3.24)$ that

$$
\mathcal{A}_{k} F_{k}^{2}-\mathcal{B}_{k} F_{k}^{1}=0, \quad c_{1} F_{k}^{1}+c_{2} F_{k}^{2}=G_{k}, \quad k=1,2,4 .
$$

Assuming that $\mathcal{A}_{k}$ is nonsingular we obtain $F_{k}^{2}=\mathcal{A}_{k}^{-1} \mathcal{B}_{k} F_{k}^{1}$, and hence $c_{1} F_{k}^{1}+$ $c_{2} \mathcal{A}_{k}^{-1} \mathcal{B}_{k} F_{k}^{1}=G_{k}$. It follows that

$$
F_{k}^{1}=\left(c_{1}+c_{2} \mathcal{A}_{k}^{-1} \mathcal{B}_{k}\right)^{-1} G_{k}=\left(c_{1} \mathcal{A}_{k}+c_{2} \mathcal{B}_{k}\right)^{-1} \mathcal{A}_{k} G_{k}
$$

if $c_{1} \mathcal{A}_{k}+c_{2} \mathcal{B}_{k}$ is nonsingular. The last condition holds if $\left(c_{1}, c_{2}\right)$ is not contained in a set of Lebesgue measure zero in $\mathbb{R}^{2}$, which may depend on $\alpha_{1}, \alpha_{2}, N$. We have similar relations if $\mathcal{B}_{k}$ is nonsingular.

In case $k=3$, we obtain $\mathcal{A}_{3} F_{3}^{2}-\mathcal{B}_{3} F_{3}^{1}=-\varepsilon\left(F_{4}^{2}-\varepsilon_{0} F_{4}^{1}\right)$ instead of $\mathcal{A}_{k} F_{k}^{2}-\mathcal{B}_{k} F_{k}^{1}=0$. A simple computation yields that

$$
F_{3}^{1}=\left(c_{1} \mathcal{A}_{3}+c_{2} \mathcal{B}_{3}\right)^{-1} \mathcal{A}_{3} G_{3}+\varepsilon c_{2}\left(c_{1} \mathcal{A}_{3}+c_{2} \mathcal{B}_{3}\right)^{-1}\left(F_{4}^{2}-\varepsilon_{0} F_{4}^{1}\right) .
$$

By taking the union of all exceptional sets of $\left(c_{1}, c_{2}\right)$ with $\alpha_{1}, \alpha_{2}$ and $N$ in the set of nonnegative integers such that $\alpha_{1}+\alpha_{2}+N \geq 2$, we see that there exists a unique formal power series solution $f^{j}(x)(j=1,2)$ of $(3.23)$, provided $\left(c_{1}, c_{2}\right)$ is not in an exceptional set of Lebesgue measure zero.

We will show the convergence of $f^{j}(x)(j=1,2)$. It is sufficient to show the convergence of $f^{1}(x)$. Let $c_{1} \mathcal{A}_{k}^{0}+c_{2} \mathcal{B}_{k}^{0}$ be the diagonal part of 
$c_{1} \mathcal{A}_{k}+c_{2} \mathcal{B}_{k},(k=1, \ldots, 4)$. In view of (3.25) we will show the following estimate: if $\left(c_{1}, c_{2}\right)$ is not contained in a set of Lebesgue measure zero such that $c_{1}<0, c_{2}<0$, then there exist $K_{1}$ and $K_{2}>0$ such that

$$
\begin{aligned}
\| c_{1} \mathcal{A}_{k}^{0}+ & c_{2} \mathcal{B}_{k}^{0} \| \geq K_{1}+K_{2}\left(\alpha_{1}+\alpha_{2}+N\right), \\
& \forall\left(\alpha_{1}, \alpha_{2}, N\right) \in \mathbb{Z}_{+}^{3}(2), k=1,2,3,4 .
\end{aligned}
$$

Since the proof is similar we consider the case $k=1$. we have

$$
c_{1} \mathcal{A}_{1}^{0}+c_{2} \mathcal{B}_{1}^{0}=\left(c_{1} \alpha_{1}-c_{1}+\left(c_{1}+c_{2}\right) \alpha_{2}+N\left(c_{1} \nu+c_{2} \mu\right)\right) I d
$$

Clearly the right-hand side has the estimate (3.26). If we have the Poincaré type estimate (3.26), then it is easy to see that the formal solution $f^{1}(x)$ converges in some neighborhood of the origin. This ends the proof.

Proof of Proposition 3.3: Let $g$ be the convergent power series defined by $g_{\alpha ; k}=0$ for $k=1,2,3$ and all $\alpha \in \mathbb{Z}_{+}^{4}(2) ; g_{\alpha_{1}, \alpha_{2}, \alpha_{3}, \alpha_{4} ; 4}=0$ if $\alpha_{4} \geq 1$; $g_{\left(\alpha_{1}, \alpha_{2}, N, 0\right) ; 4}=1$ for $\left(\alpha_{1}, \alpha_{2}, N, 0\right) \in \mathbb{Z}_{+}^{4}(2)$. We want to solve $L_{B} v=g$. Let $\lambda_{j}$ be the eigenvalues of $B$. By the same argument as in the proof of Theorem 3.1 we have the formula (3.19). Then we have

$$
v_{\left(\alpha_{1}, \alpha_{2}, 0, N\right) ; 4}=\left(-\varepsilon_{1}\right)^{N}\left(\lambda_{1} \alpha_{1}+\lambda_{2} \alpha_{2}+\lambda_{3}(N-1)\right)^{-N-1} N !
$$

for all $\left(\alpha_{1}, \alpha_{2}, N\right) \in \mathbb{Z}_{+}^{3}(2)$. The condition (3.5) implies that, for every $\tau<\tau_{0}$ we can find a constant $C>0$ and subsequence $\left\{\left(\alpha_{1, k}, \alpha_{2, k}, N_{k}\right)\right\}_{k=1}^{\infty}$ such that

$$
\left|\left(\lambda_{1} \alpha_{1, k}+\lambda_{2} \alpha_{2, k}+\lambda_{3}\left(N_{k}-1\right)\right)^{-1}\right| \geq C N_{k}^{\tau}, \quad \forall k \in \mathbb{N}
$$

Therefore, by (3.28)

$$
\left|v_{\left(\alpha_{1, k}, \alpha_{2, k}, 0, N_{k}\right) ; 4}\right| \geq\left(C\left|\varepsilon_{1}\right|\right)^{N_{k}} N_{k}^{N_{k} \tau} N_{k} !, \quad k \in \mathbb{N}, \alpha_{1} \in \mathbb{Z}_{+}(2) .
$$

Because $\varepsilon_{1} \neq 0, \tau<\tau_{0}$ and $1 / 2 \leq \tau_{0}$, (3.29) and Stirling's formula, $N$ ! $\geq$ $C^{N} N^{N}, \forall N \in \mathbb{Z}_{+}$lead to the assertion. This ends the proof of Proposition 3.3 .

We will briefly mention the general case $d$-actions. We suppose that there exist $j, 1 \leq j \leq m$ and $\ell_{0}, 1 \leq \ell_{0} \leq d$ such that $A_{j}^{\ell_{0}}$ in (1.7) admits only one dimensional eigenspace, i.e., the geometric multiplicity of $\lambda_{j}^{\ell}$ is one. 
For a positive integer $r$ we define the $r$ square nilpotent matrix $N_{r}$ by

$$
N_{r}=\left(\begin{array}{ccccc}
0 & 1 & 0 & \ldots & 0 \\
0 & 0 & 1 & \ldots & 0 \\
\vdots & \vdots & \ddots & \ddots & \vdots \\
0 & 0 & 0 \ldots & 0 & 1 \\
0 & 0 & 0 \ldots & 0 & 0
\end{array}\right)
$$

By assumption we have

$$
A_{j}^{\ell_{0}}=\lambda_{j}^{\ell_{0}} I d+\varepsilon N_{s_{j}}, \quad \varepsilon \neq 0 .
$$

By the explicit description of the centralizers of matrices (cf. [15]) all other matrices have the following form

$$
A_{j}^{\ell}=\lambda_{j}^{\ell} I d+\sum_{k=1}^{s_{j}-1} \varepsilon_{k}^{\ell j}\left(N_{s_{j}}\right)^{k} \quad \varepsilon_{k}^{\ell j} \in \mathbb{C}, k=1, \ldots, s_{j}-1 .
$$

We also suppose that there exists a sequence $\alpha^{\ell} \in \mathbb{Z}_{+}^{m}(2), \ell \in \mathbb{N}$ and a positive number $c_{0}$ such that $\left|\alpha^{\ell}\right| \rightarrow \infty(\ell \rightarrow \infty)$ and

$$
0<\omega\left(\alpha^{\ell}\right) \leq c_{0}, \quad \ell \in \mathbb{N}
$$

We have

Theorem 3.5 Assume that (3.31) and (3.33) hold. Moreover, suppose that simultaneously nonresonant condition (1.17) is satisfied. Then, one can find an $\left(f_{1}, \ldots, f_{d}\right) \in\left(\mathbb{C}_{2}^{n}\{x\}\right)^{d}$ satisfying (3.2) such that $v=L_{A}^{-1} f$ is not contained in the set $\bigcup_{1 \leq \sigma<2} G_{2}^{\sigma}\left(\mathbb{C}^{n}\right)$.

\section{Sternberg's theorem for commuting vector fields}

The results in section 2 imply that, if a cone condition holds, then simultaneous linearization of a system with a Jordan block is reduced essentially to the Poincaré-Dulac theorem for a single vector field in an analytic category. On the other hand, in view of the results in section 3, the reduction seems impossible if the cone condition is violated. 
In this section we shall illustrate that the situtation is completely different in a smooth category. We consider two commuting vector field in $\mathbb{R}^{4}$ which are in a Siegle domain and only one of the two has a linear part with nontrivial Jordan block. Obviously, the system does not satisfy a cone condition. We will show that they are simultaneously linearizable in $C^{k}$ for every $k \geq 1$.

Let $X(y)$ and $Y(y)$ be commuting $C^{\infty}$ vector fields with the common singular point at the origin $0 \in R^{4}$. Suppose that $\nabla X(0)=A, \nabla Y(0)=B$, where

$$
\begin{gathered}
A=\left(\begin{array}{cccc}
1 & 0 & 0 & 0 \\
0 & 1 & 0 & 0 \\
0 & 0 & -\nu & 0 \\
0 & 0 & 0 & -\nu
\end{array}\right), \\
B=\left(\begin{array}{cccc}
0 & 0 & 0 & 0 \\
0 & 1 & 0 & 0 \\
0 & 0 & -\mu & \varepsilon \\
0 & 0 & 0 & -\mu
\end{array}\right), \quad \varepsilon \neq 0 .
\end{gathered}
$$

We assume that the cone condition is violated, namely, (cf. Example 4.1)

$$
\nu>\mu>0, \nu \in \mathbb{R} \backslash \mathbb{Q} \text {. }
$$

We also note that the irrationality of $\nu$ implies that $X$, and hence the pair $(X, Y)$ is nonresonant. Then we have

Theorem 4.1 Suppose that the conditions (4.34), (4.35) and (4.36) are verified. Let $m \geq 1$ be an integer. Then there exists a $C^{m}$ change of the variables $y=u(x)=x+v(x), v(0)=0, \nabla v(0)=0$ near the origin which transforms both $X$ and $Y$ to their linear parts.

We need to prepare lemmas in order to prove our theorem. In view of Sternberg's theorem we assume, without loss of generality, that $X$ is linear, i.e.

$$
X v(y)=\langle\nabla v(y), A y\rangle .
$$

Let $R(y)=\left(R_{1}(y), R_{2}(y), R_{3}(y), R_{4}(y)\right)$ be the nonlinear part of $Y$

$$
Y f(y)=\langle\nabla f(y), B y+R(y)\rangle .
$$


Suppose that the change of variables $y=u(x)=x+v(x), v(0)=0, \nabla v(0)=$ 0 linearizes both the vector fields $X$ and $Y$. Then we can easily see that $v(x)$ satisfies the system of homology equations

$$
\langle\nabla v(x), B x\rangle-B v=R(x+v(x)),
$$

and

$$
\langle\nabla v(x), A x\rangle-A v=0 .
$$

We write $x=\left(x_{1}, x_{2}, x^{\prime \prime}\right)$ and $z=\left(z_{1}, z^{\prime}\right)$. Let $c_{1}>0$ and $0<c_{2} \leq 1$ be constants. Then we define

$$
\begin{gathered}
\Omega=\left\{x^{\prime}=\left(x_{2}, x_{3}, x_{4}\right)=\left(x_{2}, x^{\prime \prime}\right) \in \mathbb{R}^{3} ;\left|x_{2}\right|^{\nu}\left|x^{\prime \prime}\right|<c_{1},\left|x^{\prime \prime}\right|<c_{2}\right\}, \\
\Omega_{1}=\left\{x_{1} \in \mathbb{R} ;\left|x_{1}\right|<1\right\} \times \Omega .
\end{gathered}
$$

Then we have

Lemma 4.2 Let $k=\infty$ or $k \geq 1$ be an integer. Let $L$ be given by

$$
L=\sum_{j=1}^{2} x_{j} \partial_{x_{j}}-\nu \sum_{k=3}^{4} x_{k} \partial_{x_{k}} .
$$

Then a $C^{k}$ solution of

$$
L f(x)-f(x)=0, \quad x=\left(x_{1}, x_{2}, x_{3}, x_{4}\right) \in \Omega_{1},
$$

(respectively,

$$
\left.L w(x)+\nu w(x)=0 \quad x=\left(x_{1}, x_{2}, x_{3}, x_{4}\right) \in \Omega_{1}\right)
$$

is given by

$$
f(x)=x_{1} \varphi_{ \pm}\left(\frac{x_{2}}{x_{1}}, x_{3}\left|x_{1}\right|^{\nu}, x_{4}\left|x_{1}\right|^{\nu}\right), \quad \text { for } \pm x_{1}>0,
$$

or

$$
f(x)=x_{2} \varphi_{ \pm}\left(\frac{x_{2}}{x_{1}}, x_{3}\left|x_{1}\right|^{\nu}, x_{4}\left|x_{1}\right|^{\nu}\right), \quad \text { for } \pm x_{1}>0,
$$

(respectively, by

$$
\left.w(x)=\left|x_{1}\right|^{-\nu} \psi_{ \pm}\left(\frac{x_{2}}{x_{1}}, x_{3}\left|x_{1}\right|^{\nu}, x_{4}\left|x_{1}\right|^{\nu}\right), \quad \text { for } \pm x_{1}>0\right),
$$

where $\varphi_{ \pm}(z) \in C^{k}(\Omega)$ (respectively $\psi_{ \pm}(z) \in C^{k}(\Omega)$.) 
Proof. We apply the method of characteristics to the equations (4.43) and (4.44). Then we obtain the representations (4.45) and (4.47) for some $\varphi_{ \pm}$in $\pm x_{1}>0$.

By the commutativity we see that every component of $v=R(x)=$ $\left(R_{1}, \ldots, R_{4}\right)$ satisfies either (4.43) or (4.44). Hence, by Lemma 4.1 we have

$$
\begin{aligned}
& R_{j}(x)=x_{j} \Psi_{ \pm}^{j}\left(\frac{x_{2}}{x_{1}}, x_{3}\left|x_{1}\right|^{\nu}, x_{4}\left|x_{1}\right|^{\nu}\right), \quad \text { for } \pm x_{1}>0, j=1,2, \\
& R_{j}(x)=\left|x_{1}\right|^{-\nu} \Psi_{ \pm}^{j}\left(\frac{x_{2}}{x_{1}}, x_{3}\left|x_{1}\right|^{\nu}, x_{4}\left|x_{1}\right|^{\nu}\right), \text { for } \pm x_{1}>0, j=3,4
\end{aligned}
$$

for some functions $\Psi_{ \pm}^{j}$. In the following we will cut off $R_{j}(x)$ with a smooth function being identically equal to 1 in some neighborhood of the origin and with support contained in a small neighborhhood of the origin, which we give in the proof of Theorem 4.1. For the sake of simplicity, we denote the modified $R_{j}(x)$ with the same letter. We set

$$
z_{1}=x_{2} / x_{1}, z_{2}=x_{3}\left|x_{1}\right|^{\nu}, z_{3}=x_{4}\left|x_{1}\right|^{\nu} .
$$

For every $x_{1} \neq 0$, we define $\Psi_{ \pm}^{j}(z)$ by (4.48) and (4.49), namely

$$
\begin{aligned}
& \Psi_{ \pm}^{j}(z)=x_{j}^{-1} R_{j}\left(x_{1}, x_{1} z_{1},\left|x_{1}\right|^{-\nu} z_{2},\left|x_{1}\right|^{-\nu} z_{3}\right), \text { for } \pm x_{1}>0, j=1,2 \\
& \Psi_{ \pm}^{j}(z)=\left|x_{1}\right|^{\nu} R_{j}\left(x_{1}, x_{1} z_{1},\left|x_{1}\right|^{-\nu} z_{2},\left|x_{1}\right|^{-\nu} z_{3}\right), \text { for } \pm x_{1}>0, j=3,4 .
\end{aligned}
$$

We can easily see that $\Psi_{ \pm}^{j} \in C^{\infty}\left(\mathbb{R}_{z}^{3}\right)(j=1,2,3,4)$.

By (4.40) and simple computations we see that every component of $v(x)=$ $\left(v_{1}(x), \ldots, v_{4}(x)\right)$ satisfies either (4.43) or (4.44). It follows from Lemma 4.1 that every component of $v$ has an expression

$$
v_{j}(x)=x_{j} \varphi_{ \pm}^{j}\left(\frac{x_{2}}{x_{1}}, x_{3}\left|x_{1}\right|^{\nu}, x_{4}\left|x_{1}\right|^{\nu}\right), \quad \text { for } \pm x_{1}>0, j=1,2,
$$

and

$$
v_{j}(x)=\left|x_{1}\right|^{-\nu} \varphi_{ \pm}^{j}\left(\frac{x_{2}}{x_{1}}, x_{3}\left|x_{1}\right|^{\nu}, x_{4}\left|x_{1}\right|^{\nu}\right), \quad \text { for } \pm x_{1}>0, j=3,4,
$$

for some $\varphi_{ \pm}^{j}$. 
We substitute the transformation (4.50) and (4.53), (4.54) into (4.39), and we rewrite (4.39) as an equation of $z$ for the unknown functions $\varphi_{ \pm}^{j}(z)$ with a parameter $x_{1}$. Recalling that $v_{j}=x_{j} \varphi_{ \pm}^{j}$ and $v_{j}=\left|x_{1}\right|^{-\nu} \varphi_{ \pm}^{j}$ we obtain

$$
\begin{aligned}
& x_{2} \partial_{x_{2}} v_{1}=x_{1} z_{1} \partial_{z_{1}} \varphi_{ \pm}^{1}(z), \quad x_{3} \partial_{x_{3}} v_{1}=x_{1} z_{2} \partial_{z_{2}} \varphi_{ \pm}^{1}(z), \\
& x_{4} \partial_{x_{4}} v_{1}=x_{1} z_{3} \partial_{z_{3}} \varphi_{ \pm}^{1}(z), \quad x_{4} \partial_{x_{3}} v_{1}=x_{1} z_{3} \partial_{z_{2}} \varphi_{ \pm}^{1}(z)
\end{aligned}
$$

and we have similar relations for $v_{2}=x_{2} \varphi_{ \pm}^{2}(x)$ and $v_{j}=\left|x_{1}\right|^{-\nu} \varphi_{ \pm}^{j}(x)$. In fact we have

$$
\begin{aligned}
& \left\langle\nabla v_{1}(x), B x\right\rangle=x_{1} \mathcal{L} \varphi_{ \pm}^{1}(z), \quad \text { for } \pm x_{1}>0 \\
& \left\langle\nabla v_{2}(x), B x\right\rangle-v_{2}(x)=x_{2} \mathcal{L} \varphi_{ \pm}^{2}(z), \quad \text { for } \pm x_{1}>0 \\
& \left\langle\nabla v_{j}(x), B x\right\rangle=\left|x_{1}\right|^{-\nu} \mathcal{L} \varphi_{ \pm}^{j}(z), \quad \text { for } \pm x_{1}>0, j=3,4
\end{aligned}
$$

where

$$
\mathcal{L} f(z)=z_{1} \partial_{z_{1}} f(z)-\left(\mu z_{2}-\varepsilon z_{3}\right) \partial_{z_{2}} f(z)-\mu z_{3} \partial_{z_{3}} f(z) .
$$

We define $\varphi_{ \pm}(z)={ }^{\operatorname{tr}}\left(\varphi_{ \pm}^{1}(z), \varphi_{ \pm}^{2}(z), \varphi_{ \pm}^{3}(z), \varphi_{ \pm}^{4}(z)\right)$.

Lemma 4.3 We have the expression

$$
R_{j}(x+v(x))=x_{j} E_{ \pm}^{j}\left(z, \varphi_{ \pm}(z)\right), \quad \text { for } \pm x_{1}>0, j=1,2,
$$

where $E_{ \pm}^{j}(z, w)$ is given by

$$
E_{ \pm}^{j}(z, w)=\left(1+w_{j}\right) \Psi_{ \pm}^{j}\left(z_{1} \frac{1+w_{2}}{1+w_{1}},\left(z_{2}+w_{3}\right)\left|1+w_{1}\right|^{\nu},\left(z_{3}+w_{4}\right)\left|1+w_{1}\right|^{\nu}\right)
$$

and

$$
R_{j}(x+v(x))=\left|x_{1}\right|^{-\nu} E_{ \pm}^{j}\left(z, \varphi_{ \pm}(z)\right) \quad \text { for } \pm x_{1}>0, j=3,4,
$$

with

$E_{ \pm}^{j}(z, w)=\left|1+w_{1}\right|^{-\nu} \Psi_{ \pm}^{j}\left(z_{1} \frac{1+w_{2}}{1+w_{1}},\left(z_{2}+w_{3}\right)\left|1+w_{1}\right|^{\nu},\left(z_{3}+w_{4}\right)\left|1+w_{1}\right|^{\nu}\right)$. 
Proof. We have

$$
\begin{aligned}
\frac{x_{2}+v_{2}(x)}{x_{1}+v_{1}(x)} & =\frac{x_{2}\left(1+\varphi_{ \pm}^{2}(z)\right)}{x_{1}\left(1+\varphi_{ \pm}^{1}(z)\right)} \\
& =\frac{x_{2}}{x_{1}} \frac{1+\varphi_{ \pm}^{2}(z)}{1+\varphi_{ \pm}^{1}(z)}=z_{1} \frac{1+\varphi_{ \pm}^{2}(z)}{\left.1+\varphi_{ \pm}^{1}(z)\right)} . \\
\left(x_{3}+v_{3}(x)\right)\left|x_{1}+v_{1}\right|^{\nu} & =\left(x_{3}+\left|x_{1}\right|^{-\nu} \varphi_{ \pm}^{3}(z)\right)\left|x_{1}\right|^{\nu}\left|1+\varphi_{ \pm}^{1}(z)\right|^{\nu} \\
& =\left(x_{3}\left|x_{1}\right|^{\nu}+\varphi_{ \pm}^{3}(z)\right)\left|1+\varphi_{ \pm}^{1}(z)\right|^{\nu} \\
& =\left(z_{2}+\varphi_{ \pm}^{3}(z)\right)\left|1+\varphi_{ \pm}^{1}(z)\right|^{\nu} . \\
\left(x_{4}+v_{4}(x)\right)\left|x_{1}+v_{1}\right|^{\nu} & =\left(x_{4}+\left|x_{1}\right|^{-\nu} \varphi_{ \pm}^{4}(z)\right)\left|x_{1}\right|^{\nu}\left|1+\varphi_{ \pm}^{1}(z)\right|^{\nu} \\
& =\left(x_{4}\left|x_{1}\right|^{\nu}+\varphi_{ \pm}^{4}(z)\right)\left|1+\varphi_{ \pm}^{1}(z)\right|^{\nu} \\
& =\left(z_{3}+\varphi_{ \pm}^{4}(z)\right)\left|1+\varphi_{ \pm}^{1}(z)\right|^{\nu} .
\end{aligned}
$$

Hence, if $j=1,2$, we get

$$
\begin{aligned}
& R_{j}(x+v(x))=\left(x_{j}+v_{j}(x)\right) \\
\times & \Psi_{ \pm}^{j}\left(\frac{x_{2}+v_{2}(x)}{x_{1}+v_{1}(x)},\left(x_{3}+v_{3}(x)\right)\left|x_{1}+v_{1}\right|^{\nu},\left(x_{4}+v_{4}\right)\left|x_{1}+v_{1}(x)\right|^{\nu}\right) \\
= & x_{j}\left(1+\varphi_{ \pm}^{j}\right) \Psi_{ \pm}^{j}\left(z_{1} \frac{1+\varphi_{ \pm}^{2}}{1+\varphi_{ \pm}^{1}},\left(z_{2}+\varphi_{ \pm}^{3}\right)\left|1+\varphi_{ \pm}^{1}\right|^{\nu},\left(z_{3}+\varphi_{ \pm}^{4}\right)\left|1+\varphi_{ \pm}^{1}\right|^{\nu}\right),
\end{aligned}
$$

which yields (4.61). Similarly, we can readily prove (4.63). The proof of the lemma is complete.

Now we are ready to write explicitly the reduction of the overdetermined system for $v$ : $\left(X_{A}-A\right) v=0,\left(X_{b}-B\right) v=R(x+v(x))$ into a $4 \times 4$ system of equations for $\varphi_{ \pm}(z)$ in $z \in \Omega$ with a parameter $x_{1}$. Then the new system of semilinear homological equations for $\varphi_{ \pm}$is written as follows

$$
(\mathcal{L}-\tilde{B})\left(\varphi_{ \pm}\right)=E_{ \pm}\left(z, \varphi_{ \pm}(z)\right), \quad E_{ \pm}(z, w)=\left(E_{ \pm}^{1}(z, w), \ldots, E_{ \pm}^{4}(z, w)\right)
$$

where $E_{ \pm}^{j}(z, w)$ are given by (4.62) and (4.64) and

$$
\tilde{B}=\left(\begin{array}{cccc}
0 & 0 & 0 & 0 \\
0 & 0 & 0 & 0 \\
0 & 0 & -\mu & \varepsilon \\
0 & 0 & 0 & -\mu
\end{array}\right)
$$

We prepare a lemma. 
Lemma 4.4 Let $\nu>0$ be an irrational number. Let $f(x)$ and $w(x)$ be smooth solutions of (4.43) and (4.44) in $\Omega_{1}$, respectively satisfying that

$$
\begin{aligned}
f(0) & =w(0)=0 \\
\nabla f(0) & =\nabla w(0)=0 .
\end{aligned}
$$

We cut off $f(x)$ and $w(x)$ with a smooth function being identically equal to 1 in some neighborhood of the origin and with support contained in a small neighborhhood of the origin. For the sake of simplicity we denote the modified functions with the same letter. Let $\varphi_{ \pm}(z)$ and $\psi_{ \pm}(z)$ be defined by (4.45), (4.46) and (4.47), respectively by the same way as (4.51) and (4.52). Then, for every $\alpha \in \mathbb{Z}_{+}^{3}$, we have

$$
\partial_{z}^{\alpha} \Theta\left(z_{1}, 0\right)=0, \quad \forall z=\left(z_{1}, 0\right) \in \Omega,
$$

with $\Theta=\varphi_{ \pm}$and $\Theta=\psi_{ \pm}$.

Proof. Because $\nu$ is an irrational number we can easily see, from (4.43) and (4.44) that every $f(x)$ and $w(x)$ satisfying (4.71) and (4.72) are flat at the origin, namely all derivatives $\partial_{x}^{\alpha} f(x), \partial_{x}^{\alpha} w(x)\left(\alpha \in \mathbb{N}^{4}\right)$ vanish at the origin $x=0$. Let $\Theta(z)=\varphi_{ \pm}(z)$, and set $f(x)=x_{1} \varphi_{ \pm}\left(x_{2} / x_{1}, x_{3}\left|x_{1}\right|^{\nu}, x_{4}\left|x_{1}\right|^{\nu}\right)$, $x_{1} \neq 0$. Then we have

$$
\begin{aligned}
& \partial_{x}^{\alpha^{\prime}}\left(x_{1}^{-1} f(x)\right)=\partial_{x}^{\alpha^{\prime}} \varphi_{ \pm}\left(x_{2} / x_{1}, x_{3}\left|x_{1}\right|^{\nu}, x_{4}\left|x_{1}\right|^{\nu}\right) \\
= & \left.x_{1}^{-\alpha_{2}}\left|x_{1}\right|^{\nu\left(\alpha_{3}+\alpha_{4}\right)} \partial_{z_{1}}^{\alpha_{2}} \partial_{z_{2}}^{\alpha_{3}} \partial_{z_{3}}^{\alpha_{4}} \varphi_{ \pm}(z)\right|_{z_{1}=x_{2} / x_{1}, z_{2}=x_{3}\left|x_{1}\right|^{\nu}, z_{3}=x_{4}\left|x_{1}\right|^{\nu}} .
\end{aligned}
$$

We let $x$ tend to zero so as to satisfy $x_{2} / x_{1}=z_{1}, z_{2}=x_{3}\left|x_{1}\right|^{\nu}=0$ and $z_{3}=x_{4}\left|x_{1}\right|^{\nu}=0$. Then we have

$$
\partial_{z_{1}}^{\alpha_{2}} \partial_{z_{2}}^{\alpha_{3}} \partial_{z_{3}}^{\alpha_{4}} \varphi_{ \pm}\left(z_{1}, 0,0\right)=\lim _{x \rightarrow 0} x_{1}^{\alpha_{2}}\left|x_{1}\right|^{-\nu\left(\alpha_{3}+\alpha_{4}\right)} \partial_{x}^{\alpha^{\prime}}\left(x_{1}^{-1} f\left(x_{1}, x_{2}, 0,0\right)\right)=0,
$$

because $f(x)$ is flat at the origin. The other cases will be proved similarly.

Remark. Let $\varphi_{ \pm}(z) \in C^{k}(\Omega)$ be given. Assume that (4.73) is satisfied for $\Theta=\varphi_{ \pm}$up to some finite $|\alpha|$. Then the function $f(x)$ defined by (4.45) gives a finitely smooth solution of (4.43) if $\nu$ is an irrational number. Indeed, the finite smoothness at $x_{1}=0$ follows from the argument of Lemma 4.4. 
In order to solve (4.69) we introduce a function space. Let $N \geq 1$ and $k \leq N$ be integers. Let $0<c_{2}^{\prime}<c_{2} \leq 1$ be a constant. Then we define

$$
\begin{aligned}
\|V\|_{k ; N} & =\sup _{z \in \mathbb{R}^{3}, 0<\left|z^{\prime}\right| \leq c_{2}^{\prime}} \sum_{|\alpha| \leq k}\left|z^{\prime}\right|^{|\alpha|}\left|\partial_{z}^{\alpha}\left(\left|z^{\prime}\right|^{-N} V(z)\right)\right|, \\
|V(z)| & =\left(\sum_{j=1}^{3}\left|V_{j}(z)\right|^{2}\right)^{1 / 2}, \quad V(z)=\left(V_{1}(z), V_{2}(z), V_{3}(z)\right) .
\end{aligned}
$$

The set of all $C^{k}$ functions $V(z)$ such that $\|V\|_{k, N}<\infty$ is a Banach space $B_{k ; N}$ with the norm $\|\cdot\|_{k ; N}$. Then we have

Lemma 4.5 i) For any integers $k \geq 0$ and $0 \leq \ell \leq N$, there exists a constant $C_{k, N}>0$ such that

$$
\|u\|_{k ; \ell} \leq C_{k, N}\|u\|_{k ; N}, \quad \forall u \in B_{k ; N} .
$$

ii) For every $f, g \in B_{k ; N}$ we have $f g \in B_{k ; N}$ and there exists a constant $C_{k, N}>0$ such that

$$
\|f g\|_{k ; N} \leq C_{k, N}\|f\|_{k ; N}\|g\|_{k ; N}, \quad \forall f, g \in B_{k ; N} .
$$

Proof. Because $\left|z^{\prime}\right| \leq 1$, we have, for $|\alpha| \leq k$

$$
\begin{aligned}
& \left|z^{\prime}\right|^{|\alpha|} \partial^{\alpha}\left(\left|z^{\prime}\right|^{-\ell} u(z)\right)=\left|z^{\prime}\right|^{|\alpha|} \partial^{\alpha}\left(\left|z^{\prime}\right|^{N-\ell}\left|z^{\prime}\right|^{-N} u(z)\right) \\
= & \left|z^{\prime}\right|^{|\alpha|} \sum_{\beta+\gamma=\alpha} \partial^{\beta}\left|z^{\prime}\right|^{N-\ell} \partial^{\gamma}\left(\left|z^{\prime}\right|^{-N} u(z)\right) \leq C_{1} \sup \left|z^{\prime}\right|^{|\gamma|}\left|\partial^{\gamma}\left(\left|z^{\prime}\right|^{-N} u(z)\right)\right|
\end{aligned}
$$

for some $C_{1}>0$. This proves i).

In order to prove ii) we have, for $|\alpha| \leq k$

$$
\begin{aligned}
& \left|z^{\prime}\right|^{|\alpha|}\left|\partial^{\alpha}\left(\left|z^{\prime}\right|^{-N} f g\right)\right| \leq \sum_{\beta+\gamma=\alpha}\left|z^{\prime}\right|^{|\beta|}\left|\partial^{\beta}\left(\left|z^{\prime}\right|^{-N} f\right)\right|\left|z^{\prime}\right||\gamma|\left|\partial^{\gamma} g\right| \\
\leq & C_{2}\|f\|_{k ; N}\|g\|_{k, 0} \leq C_{3}\|f\|_{k ; N}\|g\|_{k, N} .
\end{aligned}
$$

Here $C_{2}>0$ and $C_{3}>$ are constants. This proves ii).

Let $C$ be given by

$$
C=\left(\begin{array}{ccc}
1 & 0 & 0 \\
0 & -\mu & \varepsilon \\
0 & 0 & -\mu
\end{array}\right)
$$


Then we define the operator $Q$ by

$$
Q V=-\int_{0}^{\infty} e^{-t \tilde{B}} V\left(e^{t C} z\right) d t, \quad V=\left(V_{1}, \ldots, V_{4}\right)=\left(\varphi_{ \pm}^{1}, \varphi_{ \pm}^{2}, \varphi_{ \pm}^{3}, \varphi_{ \pm}^{4}\right) .
$$

We can easily see that $U=Q V$ gives the solution of $(\mathcal{L}-\tilde{B}) U=V$. Then we have

Lemma 4.6 Let the integers $k$ and $N$ satisfy that $0 \leq k<N-\mu$ and $\mu(k+1-N)+k<0$. Then there exists $C_{k, N}(\Omega)>0$ such that

$$
\|Q V\|_{k ; N} \leq C_{k, N}(\Omega)\|V\|_{k ; N}, \quad \forall V \in B_{k ; N} .
$$

Proof. First we note that

$$
\begin{aligned}
e^{t C} z & =\left(e^{t} z_{1}, e^{-\mu t} z_{2}+e^{-\mu t} \varepsilon t z_{3}, e^{-\mu t} z_{3}\right), \\
e^{-t \tilde{B}} V & =\left(V_{1}, V_{2}, e^{\mu t}\left(V_{3}-\varepsilon t V_{4}\right), e^{\mu t} V_{4}\right) .
\end{aligned}
$$

Hence we have

$$
\begin{aligned}
V\left(e^{t C} z\right) & =V\left(e^{t} z_{1}, e^{-\mu t}\left(z_{2}+\varepsilon t z_{3}\right), e^{-\mu t} z_{3}\right) \\
& =e^{-\mu N t}\left(\left(z_{2}+\varepsilon t z_{3}\right)^{2}+z_{3}^{2}\right)^{N / 2} \tilde{V}\left(e^{t C} z\right),
\end{aligned}
$$

where $\tilde{V}(\zeta)=V(\zeta) /\left|\zeta^{\prime}\right|^{N}$. It follows that the right-hand side integral of (4.82) converges, because the growing term $e^{\mu t}$ in $e^{-t B}$ can be absorbed by $e^{-\mu N t},(\mu>0)$. First we consider the case $k=0$. By (4.85) and (4.86) we have

$$
\begin{aligned}
& \|Q V\|_{0 ; N}=\sup _{z \in \mathbb{R}^{3}, 0<\left|z^{\prime}\right| \leq c_{2}^{\prime}}\left(\frac{1}{\left|z^{\prime}\right|^{N}} \int_{0}^{\infty}\left|e^{-t \tilde{B}} V\left(e^{t C} z\right)\right| d t\right) \\
\leq & \sup _{z \in \mathbb{R}^{3}, 0<\left|z^{\prime}\right| \leq c_{2}^{\prime}}\left(\frac{1}{\left|z^{\prime}\right|^{N}} \int_{0}^{\infty}(1+|\varepsilon| t) e^{\mu t}\left|V\left(e^{t C} z\right)\right| d t\right) \\
\leq & \sup \left(\frac{1}{\left|z^{\prime}\right|^{N}} \int_{0}^{\infty}(1+|\varepsilon| t) e^{\mu(1-N) t}\left(\left(z_{2}+|\varepsilon| t z_{3}\right)^{2}+z_{3}^{2}\right)^{N / 2}\left|\tilde{V}\left(e^{t C} z\right)\right| d t\right) .
\end{aligned}
$$

On the other hand we note that

$$
\left|z^{\prime}\right|^{-N}\left(\left(z_{2}+|\varepsilon| t z_{3}\right)^{2}+z_{3}^{2}\right)^{N / 2} \leq\left|z^{\prime}\right|^{-N}\left(\left|z^{\prime}\right|+|\varepsilon| t\left|z_{3}\right|\right)^{N} \leq(1+|\varepsilon| t)^{N} .
$$


In order to estimate $\tilde{V}\left(e^{t C} z\right)$ we note the following inequality

$$
e^{-\mu t}\left(\left|z_{2}+\varepsilon t z_{3}\right|^{2}+z_{3}^{2}\right)^{1 / 2} \leq\left|z^{\prime}\right|(1+|\varepsilon| t) e^{-\mu t} \leq\left|z^{\prime}\right| \leq c_{2}^{\prime},
$$

because we have $|\varepsilon|<\mu$. It follows that

$$
\left|\tilde{V}\left(e^{t C} z\right)\right| \leq \sup _{z \in \mathbb{R}^{3}, 0<\left|z^{\prime}\right| \leq c_{2}^{\prime}}|\tilde{V}(z)|
$$

It follows that the right-hand side of (4.87) is estimated in the following way

$$
\leq \sup _{z \in \mathbb{R}^{3}, 0<\left|z^{\prime}\right| \leq c_{2}^{\prime}}|\tilde{V}(z)| \int_{0}^{\infty}(1+|\varepsilon| t)^{N+1} e^{\mu(1-N) t} d t \leq C\|V\|_{0 ; N}
$$

for some $C>0$ independent of $V$. It follows that $\|Q V\|_{0 ; N} \leq C\|V\|_{0 ; N}$ for some $C>0$.

Next we will estimate the derivative $\left|z^{\prime}\right|^{|\alpha|} \partial_{z}^{\alpha}\left(\left|z^{\prime}\right|^{-N} Q V\right)$. By Leibnitz rule it is sufficient to estimate the term $\left|z^{\prime}\right|^{|\alpha|} \partial^{\gamma}\left|z^{\prime}\right|^{-N} \partial^{\alpha-\gamma}(Q V)$, where $\alpha \geq \gamma$. By simple computations, we have $\left|z^{\prime}\right|^{|\alpha|} \partial^{\gamma}\left|z^{\prime}\right|^{-N} \leq C_{1}\left|z^{\prime}\right|^{-N+|\alpha|-|\gamma|}$ for some $C_{1}>$ independent of $z^{\prime}$. On the other hand, we have

$$
\begin{aligned}
& \partial^{\alpha-\gamma}(Q V)=-\partial^{\alpha-\gamma} \int_{0}^{\infty} e^{-t \tilde{B}}\left(\left(z_{2}+\varepsilon t z_{3}\right)^{2}+z_{3}^{2}\right)^{N / 2} e^{-\mu N t} \tilde{V}\left(e^{t C} z\right) d t \\
= & -\sum_{\beta \leq \alpha-\gamma} \int e^{-t \tilde{B}-\mu N t} \partial_{z}^{\beta}\left(\left(z_{2}+\varepsilon t z_{3}\right)^{2}+z_{3}^{2}\right)^{N / 2} \partial^{\alpha-\gamma-\beta} \tilde{V}\left(e^{t C} z\right) d t .
\end{aligned}
$$

We can easily see

$$
\left|\partial_{z}^{\beta}\left(\left(z_{2}+\varepsilon t z_{3}\right)^{2}+z_{3}^{2}\right)^{N / 2}\right| \leq C_{2}(1+|\varepsilon| t)^{N}\left|z^{\prime}\right|^{N-|\beta|}
$$

for some $C_{2}>0$. If we set $\alpha-\beta-\gamma=\delta, \delta=\left(\delta_{1}, \delta_{2}, \delta_{3}\right)$, then we have

$$
\partial^{\alpha-\beta-\gamma} \tilde{V}\left(e^{t C} z\right)=e^{t \delta_{1}-\mu\left(\delta_{2}+\delta_{3}\right) t}\left(\partial_{1}^{\delta_{1}} \partial_{2}^{\delta_{2}}\left(\varepsilon t \partial_{2}+\partial_{3}\right)^{\delta_{3}} \tilde{V}\right)\left(e^{t C} z\right) .
$$


It follows that

$$
\begin{aligned}
& \left|z^{\prime}\right|^{|\alpha|} \partial^{\gamma}\left|z^{\prime}\right|^{-N}\left|\partial^{\alpha-\gamma}(Q V)\right| \\
\leq & C_{3}\left|z^{\prime}\right|^{-N+|\alpha|-|\gamma|} \sum_{\beta} \int_{0}^{\infty} e^{\mu t-\mu N t}\left|\partial_{z}^{\beta}\left(\left(z_{2}+\varepsilon t z_{3}\right)^{2}+z_{3}^{2}\right)^{N / 2}\right| \\
\times & \left|\partial^{\alpha-\gamma-\beta} \tilde{V}\left(e^{t C} z\right)\right| d t \\
\leq & C_{3}\left|z^{\prime}\right|^{-N+|\alpha|-|\gamma|} \sum_{\beta} \int_{0}^{\infty} e^{\mu t-\mu N t}(1+|\varepsilon| t)^{N+1}\left|z^{\prime}\right|^{N-|\beta|}\left|\partial^{\alpha-\gamma-\beta} \tilde{V}\left(e^{t C} z\right)\right| d t \\
\leq & C_{4} \int_{0}^{\infty} \sum_{|\xi|=|\alpha-\beta-\gamma| \leq k}\left|z^{\prime}\right|^{|\xi|}\left|\left(\partial_{z}^{\xi} \tilde{V}\right)\left(e^{t C} z\right)\right|(1+|\varepsilon| t)^{N+1+|\xi|} e^{\mu t-\mu N t+|\alpha| t} d t .
\end{aligned}
$$

In order to estimate $\left|z^{\prime}\right| \xi||\left(\partial_{z}^{\xi} \tilde{V}\right)\left(e^{t C} z\right) \mid$, we set $\zeta=e^{t C} z$. Then we have

$$
\begin{aligned}
& \left|z^{\prime}\right|^{|\xi|}\left|\left(\partial_{z}^{\xi} \tilde{V}\right)\left(e^{t C} z\right)\right|=\left|\left(e^{-t C} \zeta\right)^{\prime}\right|^{|\xi|}\left|\left(\partial^{\xi} \tilde{V}\right)(\zeta)\right| \\
\leq & e^{\mu|\xi| t}\left|\left(\partial^{\xi} \tilde{V}\right)(\zeta)\right|\left(\left(\zeta_{2}+\varepsilon t \zeta_{3}\right)^{2}+\zeta_{3}^{2}\right)^{|\xi| / 2} \\
\leq & e^{\mu k t}(1+|\varepsilon| t)^{k}\left|\zeta^{\prime}\right|^{|\xi|}\left|\left(\partial^{\xi} \tilde{V}\right)(\zeta)\right| \leq\|V\|_{k ; N} e^{\mu k t}(1+|\varepsilon| t)^{k} .
\end{aligned}
$$

By assumption we have $(1+k-N) \mu+|\alpha| \leq(1+k-N) \mu+k<0$. Hence the right-hand side integral in (4.95) converges. Therefore we see that the right-hand side of (4.95) can be estimated by $C_{5}\|V\|_{k ; N}$.

Proof of Theorem 4.1. By setting $\varphi_{ \pm}=Q V$, (4.69) is equivalent to

$$
V=E_{ \pm}(z, Q V) \text {. }
$$

We define the sequence $V_{ \pm}^{j}(j=0,1,2, \ldots)$ by

$$
V_{ \pm}^{0}=E_{ \pm}(z, 0), \quad V_{ \pm}^{1}=E_{ \pm}\left(z, Q V_{ \pm}^{0}\right)-E_{ \pm}(z, 0)
$$

and

$$
V_{ \pm}^{j+1}=E_{ \pm}\left(z, V_{ \pm}^{0}+\cdots+V_{ \pm}^{j}\right)-E_{ \pm}\left(z, V_{ \pm}^{0}+\cdots+V_{ \pm}^{j-1}\right), \quad j=1,2, \ldots
$$

Let the integers $k$ and $N$ satisfy that $0 \leq k<N-\mu$ and $\mu(k+1-N)+$ $k<0$. We will show the convergence of $\sum_{j=0}^{\infty} V_{ \pm}^{j}$. By definition we have $V_{ \pm}^{0}=E_{ \pm}(z, 0)=\Psi_{ \pm}(z)$. Next we have

$$
V_{ \pm}^{1}=E_{ \pm}\left(z, Q V_{ \pm}^{0}\right)-E_{ \pm}(z, 0)=Q V_{ \pm}^{0} \int_{0}^{1} \nabla_{w} E_{ \pm}\left(z, \tau Q V_{ \pm}^{0}\right) d \tau
$$


Let $\varepsilon^{\prime}>0$ be a small constant chosen later, and suppose that

$$
\left\|\Psi_{ \pm}\right\|_{k ; N}<\varepsilon^{\prime}, \quad\left\|\nabla \Psi_{ \pm}\right\|_{k ; N}<\varepsilon^{\prime} .
$$

Then, by Lemma 4.5 and the definition of $V_{ \pm}^{0}$ we have

$$
\left\|\tau Q V_{ \pm}^{0}\right\|_{k ; N} \leq c_{1}\left\|V_{ \pm}^{0}\right\|_{k ; N}=c_{1}\left\|\Psi_{ \pm}\right\|_{k ; N}<c_{1} \varepsilon^{\prime}
$$

for some $c_{1}>0$ independent of $\Psi_{ \pm}$.

In order to estimate $\left\|\nabla_{w} E_{ \pm}\left(\cdot, \tau Q V_{ \pm}^{0}\right)\right\|_{k ; N}$, we set $w=\left(w_{1}, \ldots, w_{4}\right)=$ $\tau Q V_{ \pm}^{0}$ and

$$
\zeta=\left(\zeta_{1}, \zeta^{\prime}\right)=\left(z_{1} \frac{1+w_{2}}{1+w_{1}},\left(z_{2}+w_{3}\right)\left|1+w_{1}\right|^{\nu},\left(z_{3}+w_{4}\right)\left|1+w_{1}\right|^{\nu}\right) .
$$

The differentiation $\partial_{z}^{\alpha}\left(\nabla_{w} E_{ \pm}\left(z, \tau Q V_{ \pm}^{0}\right)\right)$ consists of terms which are product of $\partial^{\beta} \nabla \Psi_{ \pm}(\zeta)(\alpha \geq \beta)$ and the differentiations of $w$. First, the product of differentiations of $w$ is bounded by a constant in view of (4.102). On the other hand, in order to estimate

$$
\left|z^{\prime}\right|^{|\beta|}\left|\partial^{\beta} \nabla \Psi_{ \pm}(\zeta)\right| \leq\left|z^{\prime}\right|^{|\beta|}\left|\zeta^{\prime}\right|^{-|\beta|}\left|\zeta^{\prime}\right|^{|\beta|}\left|\partial^{\beta} \nabla \Psi_{ \pm}(\zeta)\right| \leq\left|z^{\prime}\right|^{|\beta|}\left|\zeta^{\prime}\right|^{-|\beta|}\left\|\nabla \Psi_{ \pm}\right\|_{k ; N},
$$

we consider $\left|z^{\prime}\right|^{|\beta|}\left|\zeta^{\prime}\right|^{-|\beta|}$. By Lemma 4.2 we see that $w_{3}$ and $w_{4}$ can be divisable by $z_{2}^{2}+z_{3}^{2}$, respectively. By the smallness of $w$, the term $\left|z^{\prime}\right|^{|\beta|}\left|\zeta^{\prime}\right|^{-|\beta|}$ can be bounded by a constant. Hence, if $\varepsilon^{\prime}>0$ is sufficiently small, then we obtain, by the definition of $E_{ \pm}(z, w)$ in (4.69), (4.62) and (4.64),

$$
\left\|\nabla_{w} E_{ \pm}\left(\cdot, \tau Q V_{ \pm}^{0}\right)\right\|_{k ; N} \leq c_{2}\left\|\nabla \Psi_{ \pm}\right\|_{k ; N}<c_{2} \varepsilon^{\prime}
$$

for some $c_{2}>0$ independent of $\varepsilon^{\prime}$ and $\Psi_{ \pm}$.

It follows from (4.100) that

$$
\left\|V_{ \pm}^{1}\right\|_{k ; N} \leq\left\|Q V_{ \pm}^{0}\right\|_{k ; N} \int_{0}^{1}\left\|\nabla_{w} E_{ \pm}\left(z, \tau Q V_{ \pm}^{0}\right)\right\|_{k ; N} d \tau \leq c_{1} c_{2} \varepsilon^{\prime 2} .
$$

In order to show the general case, we assume that $\left\|V_{ \pm}^{j}\right\|_{k ; N} \leq c_{1}^{j} c_{2}^{j} \varepsilon^{\prime j+1}$ for $j=0,1,2, \ldots, k$. Then we have

$$
\left\|\sum_{j=0}^{k} V_{ \pm}^{j}\right\|_{k ; N} \leq \sum_{j=0}^{k} c_{1}^{j} c_{2}^{j} \varepsilon^{\prime j+1} \leq \frac{\varepsilon^{\prime}}{1-c_{1} c_{2} \varepsilon^{\prime}} .
$$


By definition we have

$$
\begin{aligned}
V_{ \pm}^{k+1} & =E_{ \pm}\left(z, Q\left(V_{ \pm}^{0}+\cdots+V_{ \pm}^{k}\right)-E_{ \pm}\left(z, Q\left(V_{ \pm}^{0}+\cdots+V_{ \pm}^{k-1}\right)\right.\right. \\
& =Q V_{ \pm}^{k} \int_{0}^{1} \nabla_{w} E_{ \pm}\left(z, Q\left(V_{ \pm}^{0}+\cdots+V_{ \pm}^{k-1}\right)+\tau Q V_{ \pm}^{k}\right) d \tau
\end{aligned}
$$

By the apriori estimate (4.104) and Lemma 4.4 the substitution in the righthand side of (4.105) is well defined. Moreover, by the same argument as in the proof of (4.103) we see that

$$
\left\|\nabla_{w} E_{ \pm}\left(z, Q\left(V_{ \pm}^{0}+\cdots+V_{ \pm}^{k-1}\right)+\tau Q V_{ \pm}^{k}\right)\right\|_{k ; N} \leq c_{2} \varepsilon^{\prime} .
$$

It follows from (4.105) that

$$
\left\|V_{ \pm}^{k+1}\right\|_{k ; N} \leq\left\|Q V_{ \pm}^{k}\right\|_{k ; N} c_{2} \varepsilon^{\prime} \int_{0}^{1} d \tau \leq c_{1}^{k+1} c_{2}^{k+1} \varepsilon^{\prime k+2}
$$

Hence we have the estimate of $V_{ \pm}^{j}$ for $j=k+1$. It follows that the series $V_{ \pm}:=\sum_{j=0}^{\infty} V_{ \pm}^{j}$ converges in $B_{k ; N}$ and $V_{ \pm}$is a solution of (4.97). We note that, by (4.104) $V_{ \pm}$satisfies the estimate $\left\|V_{ \pm}\right\|_{k ; N} \leq \varepsilon^{\prime}\left(1-c_{1} c_{2} \varepsilon^{\prime}\right)^{-1}$, and $V_{ \pm}$ is divisable by $\left|z^{\prime}\right|^{2}$.

Next we verify the smallness assumption (4.101) uniformly with respect to $x_{1} \neq 0$ in some neighborhood of $x_{1}=0$. Because the argument is similar we consider the condition $\left\|\Psi_{ \pm}\right\|_{k ; N}<\varepsilon^{\prime}$. In view of the definition of $\Psi_{ \pm}$in (4.51) and (4.52), we estimate $x_{j}^{-1} R_{j}\left(x_{1}, x_{1} z_{1},\left|x_{1}\right|^{-\nu} z_{2},\left|x_{1}\right|^{-\nu} z_{3}\right),(j=1,2)$ and $\left|x_{1}\right|^{\nu} R_{j}\left(x_{1}, x_{1} z_{1},\left|x_{1}\right|^{-\nu} z_{2},\left|x_{1}\right|^{-\nu}\right),(j=3,4)$ with $x_{1} \neq 0$ close to 0 . Because the argument is similar, we consider the case $j=1$. We have

$$
\left|z^{\prime}\right|^{|\alpha|}\left|\partial_{z}^{\alpha}\left(\left|z^{\prime}\right|^{-N} \Psi_{ \pm}^{1}(z)\right)\right|=x_{1}^{-1}\left|z^{\prime}\right|^{|\alpha|}\left|\partial_{z}^{\alpha}\left(\left|z^{\prime}\right|^{-N} R_{1}\left(x_{1}, x_{1} z_{1},\left|x_{1}\right|^{-\nu} z_{2},\left|x_{1}\right|^{-\nu} z_{3}\right)\right)\right| .
$$

By Lemma 4.4 we have that, for every positive integer $p$, the term

$$
R_{1}\left(x_{1}, x_{1} z_{1},\left|x_{1}\right|^{-\nu} z_{2},\left|x_{1}\right|^{-\nu} z_{3}\right)\left|z^{\prime}\right|^{-p}
$$

is smooth at $z=0$. Because

$$
\left|z^{\prime}\right|^{p}=\left(\left|x_{1}\right|^{\nu}\left|x_{1}\right|^{-\nu}\left|z^{\prime}\right|\right)^{p}=\left(\left|x_{1}\right|^{\nu}\left|x^{\prime \prime}\right|\right)^{p}, \quad x^{\prime \prime}=\left(x_{3}, x_{4}\right),
$$

and $\left|x^{\prime \prime}\right|$ is bounded by the support condition of $R_{j}$, the negative power $\left|z^{\prime}\right|^{-N}$ in the right-hand side of (4.106) is absorbed by $\left|z^{\prime}\right|^{p}$ if $p$ is sufficiently large. On the other hand, if the differentiation $\partial_{z}^{\alpha}$ is applied to 
$R_{1}\left(x_{1}, x_{1} z_{1},\left|x_{1}\right|^{-\nu} z_{2},\left|x_{1}\right|^{-\nu} z_{3}\right)$, then the negative power of $\left|x_{1}\right|$ appears. These terms are also uniformly bounded when $x_{1} \rightarrow 0$, because there appears positive power of $\left|x_{1}\right|$ from $\left|z^{\prime}\right|^{p}$. Because all derivatives of $R(x)$ at the origin vanish, we see that the right-hand side of (4.106) can be made arbtrarily small if we cut off $R_{(x)}$ in a sufficiently small neighborhood of the origin. This proves that we have (4.101).

We set $\varphi_{ \pm}=Q V_{ \pm} \in B_{k ; N}$, and $\varphi_{ \pm}(z)=\left(\varphi_{ \pm}^{1}(z), \varphi_{ \pm}^{2}(z), \varphi_{ \pm}^{3}(z), \varphi_{ \pm}^{4}(z)\right)$. The function $\varphi_{ \pm}$is a solution of (4.69). Then we define $v^{j}(x)(j=1,2,3,4)$ by (4.53) and (4.54). For a given integer $m$, we can easily see that $v^{j}(x)$ is a $C^{m}$ function if we take $k$ and $N$ in $B_{k ; N}$ sufficiently large. If we rewrite (4.69) with the variable $x$, then we see that $v$ is a solution of (4.39), where the nonlinear part $R$ is modified by a cutoff function. In order to show that $v$ is a solution of the original (4.39) we will show the apriori estimate of $v$. In fact, if $|x+v|<\varepsilon^{\prime \prime}$ for sufficiently small $\varepsilon^{\prime \prime}$, then $v$ is a solution of (4.39). By Lemma 4.6 and the uniform estimate of $V_{ \pm}$in $x_{1}$ we know that $\phi_{ \pm}^{1}(z)$ is uniformly bounded in $z$ and $x_{1}$. It follows that $v_{1}(x)=x_{1} \phi_{ \pm}^{1}$ is arbitrarily small if $x_{1}$ is sufficiently small. Similarly we can show that $v_{2}(x)=x_{2} \phi^{2}$ is small by the estimate of $V_{ \pm}$. On the other hand, we have $x_{3}+v_{3}(x)=x_{3}+\left|x_{1}\right|^{-\nu} \phi_{ \pm}^{3}(z)$. Because $\phi_{ \pm}^{3}$ is divisable by $\left|z^{\prime}\right| 2$ and $\left|z^{\prime}\right|=\left|x_{1}\right|^{\nu}\left|x^{\prime \prime}\right|$, by Lemma 4.4 we see that $\left|x_{3}+v_{3}(x)\right|<\varepsilon^{\prime \prime}$ uniformly in $x_{1}$. Similarly we can show the same estimate for $x_{4}+v_{4}$. Therefore we see that $v$ is a solution of (4.39). This completes the proof.

Acknowledgements. This work was done when the first author stayed in RIMS, Kyoto University and the second author stayed in the University of Cagliari. The first author thanks Prof. T. Kawai for the invitation to RIMS, Kyoto University and for the excellent conditions for the research on the subject of the paper. The second author expresses the sincere gratitude to the University of Cagliari for the support in the beginning of this research. The authors also acknowledge useful discussions on problems related to the paper with S. Marmi, R. Perez Marco and L. Stolovitch.

\section{References}

[1] M. Abate, Diagonalization of nondiagonalizable discrete holomorphic dynamical systems. Amer. J. Math. 122 , 757-781 (2000). 
[2] V. I. Arnold, Geometrical Methods in the Theory of Ordinary Differential Equations. Springer, New York - Heidelberg - Berlin, 1983.

[3] A.D. Bruno, The analytic form of differential equations, Tr. Mosk. Mat. O-va 25, 119-262 (1971) and 26, 199-239 (1972) (in Russian); see also Trans. Mosc. Math. Soc. 25, 131-288 (1971) and 26, 199-239 (1972).

[4] A.D. Bruno and S. Walcher, Symmetries and convergence of normalizing transformations, J. Math. Anal. Appl. 183, 571-576 (1994).

[5] T. Carletti, Exponentially long time stability for non-linearizable analytic germs of $\left(C^{n}, 0\right)$, Ann Inst. Fourier (Grenoble) 54 (2004), 4, 9891004 .

[6] T. Carletti and S. Marmi, Linearization of analytic and non-analytic germs of diffeomorphisms of $(C, 0)$, Bull. Soc. Math. France, 128, 69-85 (2000).

[7] K.T. Chen, Diffeomorphisms: $C^{\infty}$-realizations of formal properties, Amer. J. Math.87, 140-157 (1965).

[8] G. Cicogna and G. Gaeta, Symmetry and perturbation theory in nonlinear dynamics. Lecture Notes in Physics. New Series m: Monographs, 57. Springer-Verlag, Berlin, 1999.

[9] G. Cicogna and S. Walcher, Convergence of normal form transformations: the role of symmetries. Symmetry and perturbation theory. Acta Math. Appl. 70 , 95-111 (2002).

[10] F. Dumortier and R. Roussarie, Smooth linearization of germs of $R^{2}$ actions and holomorphic vector fields, Ann. Inst. Fourier, Grenoble, 30 (1), 31-64 (1980).

[11] R. De La Llave, A tutorial on KAM theory. to appear in Proc. of 1999 Summer Research Institute on Smooth Ergodic Theory and Applications, July 26-Augsut 13, 1999, Univ. of Washington, Seattle.

[12] D. DeLatte and T. Gramchev, Biholomorphic maps with linear parts having Jordan blocks: Linearization and resonance type phenomena. Math. Phys. Electron. J., 8, paper n. 2, 1-27 (2002). 
[13] D. Dickinson, T. Gramchev and M. Yoshino, Perturbations of vector fields on tori: resonant normal forms and Diophantine phenomena. Proc. Edinb. Math. Soc., II. Ser.45:3, 731-159 (2002).

[14] J. Ecalle and B. Vallet, Correction and linearization of resonant vector fields and diffeomorphisms. Math. Z. 229, 249-318 (1998).

[15] F.R. Gantmacher, The theory of matrices, Vols. 1, 2. Chelsea Publishing Co., New York, 1959.

[16] T. Gramchev, On the linearization of holomorphic vector fields in the Siegel Domain with linear parts having nontrivial Jordan blocks, S. Abenda, G. Gaeta and S. Walcher eds, Symmetry and perturbation theory, Cala Gonone, 16-22 May 2002, World Scientific, Singapore (2003), 106-115.

[17] T. Gramchev and M. Yoshino, Rapidly convergent iteration method for simultaneous normal forms of commuting maps. Math. Z., 231, 745-770 (1999).

[18] M. Hibino, Divergence Property of Formal Solution for First Order Linear Partial Differential Equations, Publ. RIMS, Kyoto Univ. 35, 893919, (1999).

[19] F. Ichikawa, Finitely determined singularities of formal vector fields, Inv. Math. 66, 199-214 (1982).

[20] Y. Il'yashenko: Divergence of series reducing an analytic differential equation to linear form at a singular point. Funct. Anal. and Appl. 13, 227-229 (1979).

[21] A. Katok and S. Katok, Higher cohomology for Abelian groups of toral automorphisms. Ergodic Theory Dyn. Syst. 15: 3, 569-592 (1995).

[22] J. Moser, On commuting circle mappings and simultaneous Diophantine approximations. Math. Z. 205, 105-121 (1990).

[23] R. Pérez Marco, Non linearizable holomorphic dynamics having an uncountable number of symmetries. Inv. Math. 119, 67-127 (1995).

[24] R. Pérez Marco, Total convergence or small divergence in small divisors. Commun. Math. Phys. 223:3, 451-464 (2001). 
[25] R. Rousssarie, Modéles locaux de champs et de formes, Astérisque 30, (1975).

[26] W. Schmidt, Diophantine approximation. Lect. Notes in Mathematics, 785, Springer Verlag, Berlin - Heidelberg - New York 1980.

[27] S. Sternberg: The structure of local homeomorphisms II, III, Amer. J. Math 80, 623-632 and 81, 578-604 (1958).

[28] L. Stolovitch: Singular complete integrability. Publ. Math. I.H.E.S., 91, 134-210 (2000).

[29] S. Walcher, On convergent normal form transformations in presence of symmetries. J. Math. Anal. Appl. 244, 17-26 (2000).

[30] J.-C. Yoccoz: A remark on Siegel's theorem for nondiagonalizable linear part, manuscript, 1978, see also Théorème de Siegel, nombres de Bruno e polynômes quadratic, Astérisque 231, 3-88 (1995).

[31] M. Yoshino: Simultaneous normal forms of commuting maps and vector fields. A. Degasperis, G. Gaeta eds., Symmetry and perturbation theory SPT 98, Rome 16-22 December 1998, World Scientific, Singapore, 287294 (1999).

[32] N.T. Zung: Convergence versus integrability in Poincaré-Dulac normal form. Math. Res. Lett. 9, no. 2-3, 217-228 (2002). 